\title{
Improvement of marker-based predictability of Apparent Amylose Content in japonica rice through GBSSI allele mining
}

\author{
Chiara Biselli ${ }^{1,2+}$, Daniela Cavalluzzo ${ }^{1 \dagger}$, Rosaria Perrini ${ }^{1}$, Alberto Gianinetti ${ }^{2}$, Paolo Bagnaresi ${ }^{2}$, Simona Urso ${ }^{2}$, \\ Gabriele Orasen ${ }^{1}$, Francesca Desiderio ${ }^{2}$, Elisabetta Lupotto ${ }^{3}$, Luigi Cattivelli ${ }^{2}$ and Giampiero Vale $e^{1,2^{*}}$
}

\begin{abstract}
Background: Apparent Amylose Content (AAC), regulated by the Waxy gene, represents the key determinant of rice cooking properties. In occidental countries high AAC rice represents the most requested market class but the availability of molecular markers allowing specific selection of high AAC varieties is limited.

Results: In this study, the effectiveness of available molecular markers in predicting AAC was evaluated in a collection of 127 rice accessions (125 japonica ssp. and 2 indica ssp.) characterized by AAC values from glutinous to $26 \%$. The analyses highlighted the presence of several different allelic patterns identifiable by a few molecular markers, and two of them, i.e., the SNPs at intron 1 and exon 6, were able to explain a maximum of $79.5 \%$ of AAC variation. However, the available molecular markers haplotypes did not provide tools for predicting accessions with AAC higher than 24.5\%. To identify additional polymorphisms, the re-sequencing of the Waxy gene and $1 \mathrm{kbp}$ of the putative upstream regulatory region was performed in 21 genotypes representing all the AAC classes identified. Several previously un-characterized SNPs were identified and four of them were used to develop dCAPS markers.

Conclusions: The addition of the SNPs newly identified slightly increased the AAC explained variation and allowed the identification of a haplotype almost unequivocally associated to AAC higher than $24.5 \%$. Haplotypes at the waxy locus were also associated to grain length and length/width (L/W) ratio. In particular, the SNP at the first intron, which identifies the $W x^{a}$ and $W x^{b}$ alleles, was associated with differences in the width of the grain, the L/W ratio and the length of the kernel, most likely as a result of human selection.
\end{abstract}

Keywords: Apparent amylose content; Grain shape characters; Marker-Assisted Selection (MAS); Molecular markers; Re-sequencing; Rice (Oryza sativa L.)

\section{Background}

Rice (Oryza sativa L.) is the most important staple food for over half of the world population. Rice quality is primarily influenced by starch which is composed of two polysaccharides: amylose and amylopectin. The percentage of amylose on total starch, measured as Apparent Amylose Content (AAC), is the key determinant of rice

\footnotetext{
*Correspondence: giampiero.vale@entecra.it

${ }^{\dagger}$ Equal contributors

'Rice Research Unit, CRA-Consiglio per la Ricerca e la Sperimentazione in Agricoltura, S.S. 11 to Torino, Km 2,5, 13100 Vercelli, Italy

${ }^{2}$ Genomics Research Centre, CRA-Consiglio per la Ricerca e la

Sperimentazione in Agricoltura, Via S. Protaso 302, 29017 Fiorenzuola d'Arda,

Piacenza, Italy

Full list of author information is available at the end of the article
}

cooking properties. High amylose varieties, like risotto varieties, cook dry with firm and separate grain; while low amylose cultivars (cvs.) are tender, glossy and cohesive after cooking. Suggested classification of amylose content identified classes as waxy $(0-5 \%)$, very low (5$12 \%)$, low (12-20\%), intermediate (20-25\%), and high (25-33\%), even considering that commercially rice is classified by amylose content as either low (less than $20 \%$ amylose), medium (21-25\%) and high (26-33\%) (Juliano 1992; Suwannaporn et al. 2007).

Over the past several decades, various methods have been reported for the determination of amylose content, including iodine binding, near infrared spectroscopy, sizeexclusion chromatography and most recently, asymmetric

\section{空}

(c) 2014 Biselli et al.; licensee Springer. This is an Open Access article distributed under the terms of the Creative Commons Attribution License (http://creativecommons.org/licenses/by/2.0), which permits unrestricted use, distribution, and reproduction in any medium, provided the original work is properly cited. 
field flow fractionation (Juliano 1971; Wesley et al. 2003; Ward et al. 2006; Chiaramonte et al. 2012). However, none of these methods is cost-effective in terms of high throughput screening (Caffagni et al. 2013) and the iodine binding represents the only one which has been validated for routine use (Fitzgerald et al. 2009). The utilization of marker-assisted selection (MAS) could overcome the shortcomings of amylose content measurements and, considering its lower cost and higher throughput, can be applied as a selection tool in the early phases of the breeding, while AAC direct analysis would require seeds setting. In addition, DNA markers for AAC also allow homozygous and heterozygous plants to be readily distinguished and often provides a more definitive way of classifying Granule-Bound Starch Synthase (GBSS) alleles compared to AAC assays since it avoids complications such as modifier genes, cytoplasmic factors, as well as environmental effects such as differences in temperature during grain development (McKenzie and Rutger 1983; Asaoka et al. 1985; Kumar and Khush 1987).

Amylose synthesis in developing seeds is primarily regulated by the $\operatorname{Waxy}(W x)$ gene which encodes the Granule-Bound Starch Synthase I (GBSSI) enzyme and the level of grain amylose is directly associated to the amount of GBSSI in the endosperm (Mikami et al. 2008). The Waxy gene is located on chromosome 6 and consists of 13 exons and 12 introns. Two wild type alleles, $W x^{a}$, primarily found in indica subspecies, and $W x^{b}$, mainly found in japonica subspecies, have been found to predominate at the waxy locus for high and low AAC respectively (Dobo et al. 2010). The difference between the two alleles is related to the presence in $W x^{b}$ of a $G$ to $T$ Single Nucleotide Polymorphism (SNP) at the 5 ' splice site of the first 1,124 bp long intron, localized 1,164 bp upstream the start codon (haplotype AGTT instead of AGGT). Most of the waxy and low AAC cvs. screened so far carry this polymorphism which results, for $W x^{b}$, in the reduction of pre-mRNA splicing efficiency and promotion of alternative splicing at cryptic sites in exon 1, leading to a decreased production of functional enzymes and causing the glutinous and low amylose phenotypes (Wang et al. 1995; Ayres et al. 1997; Bligh et al. 1998; Cai et al. 1998; Isshiki et al. 1998; Larking and Park 1999). As the SNP implies the generation of a restriction site, a Cleaved Amplified Polymorphic Sequence (CAPS) marker was developed and largely used to discriminate between high ( $\mathrm{G}$ allele) and low ( $\mathrm{T}$ allele) amylose varieties. Another diagnostic molecular marker widely utilized is the RM190 $\mathrm{CT}_{\mathrm{n}}$ microsatellite, located in the $5^{\prime} \mathrm{Un}$ translated Region (UTR) of Waxy exon 1 (Bligh et al. 1995). Seven variants of this marker are known enabling the discrimination between low, intermediate and high AAC genotypes (Ayres et al. 1997; Shu et al. 1999; Bergman et al. 2001; Tan and Zhang 2001). However, failures in detecting specific correlation between the number of RM190 repeats and an AAC group were frequently observed, suggesting that this microsatellite is simply a closely linked marker rather than the causal mutation for AAC variation (Ayres et al. 1997; Dobo et al., 2010).

The ever-increasing demand of rice varieties with higher eating quality drives to the identification of superior and novel alleles to be used in breeding programs. The allele mining approach, based on the sequencing of different alleles of a single gene in different genotypes within a specie, has been largely applied to mine the sequence diversification at the level of the key genes for quality with the aim of studying the available diversity and/or developing allele-specific molecular markers for Marker-Assisted Selection (MAS). One example in rice is the identification of 24 SNPs and one InDel mutation obtained through the comparison of the Starch Synthase II (SSII) gene sequence in 30 rice genotypes. The polymorphisms were then utilized for marker-assisted backcross breeding programs (Bao et al. 2006). Moreover, Takanokai and co-workers (2009) compared the sequence of the GS3 gene, responsible of grain size, in 54 rice cvs. and identified 86 SNPs and 20 InDels. The allele mining for the rice fragrant gene badh2 also allowed the development of diagnostic molecular markers (Shi et al. 2008).

Allele mining experiments applied to the Waxy gene led to the identification of five different allelic variants. Mikami et al. (2008) studied the allelic diversification at the $W x$ locus in Asian rice associating different alleles to grain amylose content alterations. They identified the $W x^{o p}$ allele in indica varieties from India, Nepal, Indonesia and China showing an opaque and chalky endosperm with a very low amylose content. This allele is characterized by an A to G SNP in exon 4 at position +715 from the ATG causing an Asp to Gly aminoacid change. The allele $W x^{\text {in }}$ was frequent in accessions belonging to an aromatic group and to tropical japonica which exhibited an intermediate $\mathrm{AAC}$ and is determined by a non-conservative mutation in exon 6 at position $+1,083$. The mutation alters a Tyr to Ser in the active site of the enzyme reducing its specific activity (Dobo et al. 2010). The last allele analyzed by Mikami and co-workers (2008), the $w x$ allele, is present only in waxy varieties and is characterized by a $23 \mathrm{bp} \mathrm{du}-$ plication in the second exon, $100 \mathrm{bp}$ downstream the ATG, causing a premature stop codon which inactivates the Waxy gene. A minor allele, represented by $W x^{m q}$, was identified in the low AAC rice cv. Milky Queen and was characterized by two base changes within the coding region: $\mathrm{G}$ to $\mathrm{A}$ in exon 4 and $\mathrm{T}$ to $\mathrm{C}$ in exon 5 . Each of these mutations generated missense aminoacid substitutions (Sato et al. 2002). One additional low AAC-associate allele, $W x^{h p}$, was identified by Liu et al. (2009) in a few 
low AAC Yunnan landraces. An A to G SNP occurring in exon 2 at position +497 causes an Asp to Gly substitution resulting in a reduction of the activity of GBSSI.

Following the discovery of this variability at the $W x$ locus, additional AAC class-specific molecular markers were developed. The combination of the $W x^{\text {in }}$ SNP in exon 6 and a SNP in exon 10, which consists of a C/T non-conservative SNP, with the RM190 microsatellite in exon 1 enabled the discrimination between intermediate and high AAC in a small number of genotypes (Larkin and Park 2003). More recently, Dobo and co-workers (2010) performed the same analysis increasing the number of genotypes and identified three allelic groups based on the combination of the three SNPs explaining 89.2\% of the variation in AAC among 85 US varieties and 93.8\% of the variation in 279 European accessions.

To date, the attention has been focalised in the identification of $W x$ genetic variants explaining amylose reduction in the endosperm of intermediate, low and waxy genotypes and the molecular markers used so far can discriminate only waxy, low, intermediate and high AAC. There are no markers explaining the different percentages of seed amylose within the high amylose class and further elucidations are needed to improve rice quality particularly in occidental countries where dry and firm rice is largely preferred.

In this study a collection of 127 rice accessions (125 japonica ssp. and 2 indica ssp.) was analyzed for AAC leading to the identification of the AAC classes low, intermediate and high. Effectiveness of available markers diagnostic for Apparent Amylose Content was verified and a low predictability within the high AAC class was observed. To discover new molecular markers associated to the different AACs, twenty-one genotypes representing each AAC class were selected and subjected to the re-sequencing of the waxy locus. New SNPs were identified in four high AAC accessions and used to develop new SNP-based molecular markers. Moreover, un-expected associations between grain shape characters and polymorphisms associated to the waxy locus were identified and analyzed.

\section{Results}

\section{AAC assessment in a rice germplasm collection}

A collection of 125 temperate japonica and two indica rice accessions originated from different rice cultivation areas was evaluated for AAC and four classes were identified, ranging from waxy to high AAC (Table 1). No accessions of the very low AAC class (5-12\%) were detected; high frequency was instead observed for low and intermediate amylose classes (Figure 1) while a low frequency of the high amylose class was highlighted. Accessions showing AAC higher than 20\% (60 in total) originated from very different countries (20 from USA, 11 from Italy,
4 from Portugal, 3 from Spain, 2 from France and the remaining from other countries), indicating that the trait conferring a relatively high amylose content was selected in different rice cultivation areas.

\section{Molecular markers analyses}

The germplasm collection of 127 rice accessions (Table 1) was chosen to evaluate the effectiveness of known molecular markers in predicting Apparent Amylose Content. Ten different alleles for the RM190 CTn microsatellite were identified (Table 2) but a clear relationship with the different AAC groups was observed only for some alleles (Table 1). Repeats $\mathrm{CT}_{9}, \mathrm{CT}_{10}$ and $\mathrm{CT}_{14}$ were present only in genotypes with $23-24.85 \% \mathrm{AAC}$, while $\mathrm{CT}_{11}$ and $\mathrm{CT}_{20}$ also identified accessions with AAC higher than $25 \%$. Among this AAC classes, two varieties showed unique CT alleles: $\mathrm{CT}_{13}$ for Ota (22.55\% AAC) and $\mathrm{CT}_{21}$ for Bomba (22.84\% AAC). The most frequent allele, $\mathrm{CT}_{18}$ identified in 67 accessions, represents a wide range of AAC (14.92$21.56 \%$ ). Similar results were observed for $\mathrm{CT}_{17}$ and $\mathrm{CT}_{19}$ which were associated to heterogeneous AAC intervals ranging from $15.55 \%$ to $23.27 \%$. Considering nonglutinous genotypes, the RM190 microsatellite explained the $74.9 \%$ of the AAC variation in our collection (Table 3).

For the SNP in the splicing site of the leader intron (intron 1), results confirming previous investigations were observed (Wang et al. 1995; Ayres et al. 1997; Bligh et al. 1998; Cai et al. 1998; Isshiki et al. 1998; Larking and Park 2003; Dobo et al. 2010). With only two exceptions represented by Rotundus and Antoni, all the accessions with AAC lower than $22 \%$ showed the $\mathrm{T}$ allele, while all the genotypes with AAC higher than $22 \%$ had the $\mathrm{G}$ allele (Table 1). This SNP explained a higher level of AAC variation (77.5\%) with respect to the RM190 (Table 3) and the combination of the two polymorphisms did not significantly increase the level of explained variability $(77.7 \%)$.

The presence of SNPs characterizing the different known $W x$ alleles was assessed in our collection through sequencing of exons 2, 4, 5 and 6. The $w x$ allele was identified in the waxy cv. Calmochi 101, in which the 23 bp duplication (sequence motif: ACGGGTTCCAGG GCCTCAAGCCC) in exon 2 responsible for Waxy gene inactivation was present; literature data in fact provide $\mathrm{AAC}$ values for this $\mathrm{cv}$. ranging from 0.8 to $1 \%$ (Park et al. 2007; Li et al. 2008). Allelic variation in the $W x^{i n}$ allele, characterized by a non-conservative $\mathrm{A} / \mathrm{C}$ mutation in exon 6, was observed with the A allele being detected, with few exceptions, in accessions with AAC ranging from waxy to $22 \%$ and higher than $24 \%$, and the C allele generally present in genotypes with an AAC from 22 to $24 \%$ (Table 1). No polymorphisms were identified in exons 4 and 5, representative of the $W x^{m q}$ allele and, unlike in previous observations (Dobo et al. 2010), we did 


\begin{tabular}{|c|c|c|c|c|c|c|c|c|c|c|c|c|c|c|c|c|}
\hline Accession & AAC (\%) & EU Classification & Group & Origin & $\begin{array}{c}\text { Maturity } \\
\text { time (days) }\end{array}$ & $\begin{array}{l}\text { Grain length } \\
(\mathrm{mm})^{\mathrm{a}}\end{array}$ & $\begin{array}{l}\text { Grain width } \\
\quad(\mathrm{mm})^{\mathrm{a}}\end{array}$ & $\begin{array}{l}\text { to width } \\
\text { ratio }\end{array}$ & RM190 & $\begin{array}{c}\mathrm{T} / \mathrm{G} \\
\text { intron } 1\end{array}$ & $\begin{array}{c}A / C \\
\text { exon } 6\end{array}$ & $\begin{array}{c}\text { _ } 1514 \mathrm{G} / \mathrm{T} \\
\text { promoter }\end{array}$ & $\begin{array}{r}1801 \mathrm{~T} / \mathrm{C} \\
\text { exon } 9\end{array}$ & $\begin{array}{l}2282 \mathrm{~A} / \mathrm{G} \\
\text { intron } 10\end{array}$ & $\begin{array}{l}2806 \mathrm{C} / \mathrm{T} \\
\text { intron } 12\end{array}$ & \\
\hline CALMOCHI 101 & - & Medium & Jap & USA & 158 & 5,26 & 2,97 & 1,77 & 17 & $\bar{T}$ & A & G & $\bar{T}$ & $A$ & C & \\
\hline PREVER & 14,92 & Long B & Jap & ITA & 141 & 7,22 & 2,16 & 3,34 & 18 & $\mathrm{~T}$ & A & G & $\mathrm{T}$ & A & C & \\
\hline MEJANES 2 & 15,21 & Long B & Jap & FRA & 144 & 8,24 & 2,28 & 3,61 & 18 & $\mathrm{~T}$ & A & G & $\mathrm{T}$ & A & C & \\
\hline YRL 196 & 15,55 & Medium & Jap & AUS & 142 & 5,90 & 2,61 & 2,26 & 19 & $\mathrm{~T}$ & A & G & $\mathrm{T}$ & A & C & \\
\hline DELTA & 15,60 & Long A & Jap & FRA & 139 & 8,16 & 2,90 & 2,81 & 18 & $\mathrm{~T}$ & A & G & $\mathrm{T}$ & A & C & \\
\hline LOMELLINO & 15,77 & Medium & Jap & ITA & 129 & 5,67 & 3,22 & 1,76 & 18 & $\mathrm{~T}$ & A & G & $\mathrm{T}$ & A & C & \\
\hline SOURE & 15,86 & Long A & Jap & PRT & 149 & 7,56 & 2,88 & 2,63 & 18 & $\mathrm{~T}$ & A & G & $\mathrm{T}$ & A & C & to \\
\hline CAMPINO & 15,90 & Medium & Jap & PRT & 145 & 6,19 & 3,39 & 1,83 & 18 & $\mathrm{~T}$ & A & G & $\mathrm{T}$ & A & C & S \\
\hline EUROSIS & 16,11 & Long A & Jap & ITA & 149 & 6,73 & 2,52 & 2,67 & 18 & $\mathrm{~T}$ & A & G & $T$ & A & C & \\
\hline FAMILIA 181 & 16,27 & Long A & Jap & PRT & 159 & 7,05 & 2,85 & 2,47 & 18 & $\mathrm{~T}$ & A & G & $\mathrm{T}$ & A & C & \\
\hline TAICHUNG 65 & 16,29 & Long A & Jap & TWN & 149 & 6,58 & 2,66 & 2,47 & 18 & $\mathrm{~T}$ & A & G & $\mathrm{T}$ & A & C & \\
\hline SMERALDO & 16,34 & Long A & Jap & ITA & 159 & 6,89 & 2,64 & 2,61 & 18 & $\mathrm{~T}$ & A & G & $\mathrm{T}$ & A & C & \\
\hline YRM 6-2 & 16,40 & Medium & Jap & AUS & 160 & 5,93 & 2,90 & 2,04 & 19 & $\mathrm{~T}$ & A & G & $\mathrm{T}$ & A & C & \\
\hline TIMICH 108 & 16,42 & Round & Jap & $\mathrm{ROM}$ & 127 & 4,92 & 3,13 & 1,57 & 17 & $\mathrm{~T}$ & A & G & $\mathrm{T}$ & A & C & \\
\hline GIOVANNI MARCHEITI & 16,53 & Medium & Jap & ITA & 140 & 5,76 & 3,29 & 1,75 & 18 & $\mathrm{~T}$ & A & G & $\mathrm{T}$ & A & C & \\
\hline TOPAZIO & 16,55 & Medium & Jap & PRT & 138 & 5,70 & 3,31 & 1,72 & 18 & $\mathrm{~T}$ & A & G & $\mathrm{T}$ & A & C & \\
\hline ZENA & 16,57 & Long B & Jap & ITA & 144 & 7,49 & 2,16 & 3,47 & 18 & $\mathrm{~T}$ & A & G & $\mathrm{T}$ & A & C & \\
\hline RIBE 253 & 16,71 & Long A & Jap & ITA & 149 & 6,91 & 2,88 & 2,40 & 18 & $\mathrm{~T}$ & A & G & $\mathrm{T}$ & A & C & \\
\hline PIEMONTE & 16,98 & Long A & Jap & ITA & 149 & 6,21 & 3,25 & 1,91 & 19 & $\mathrm{~T}$ & A & G & $\mathrm{T}$ & A & C & \\
\hline RPC 12 & 17,03 & Round & Jap & $\mathrm{CHN}$ & 141 & 5,01 & 3,11 & 1,61 & 18 & $\mathrm{~T}$ & A & G & $\mathrm{T}$ & A & C & \\
\hline RONCOLO & 17,06 & Medium & Jap & ITA & 144 & 5,69 & 3,22 & 1,77 & 18 & $\mathrm{~T}$ & A & G & $\mathrm{T}$ & A & C & \\
\hline SLAVA & 17,23 & Medium & Jap & $B G R$ & 144 & 6,14 & 3,38 & 1,82 & 18 & $\mathrm{~T}$ & A & G & $T$ & A & C & \\
\hline SELN 244 A6-20 & 17,29 & Medium & Jap & AUS & 154 & 5,34 & 3,17 & 1,68 & 18 & $\mathrm{~T}$ & A & G & $\mathrm{T}$ & A & C & \\
\hline BONNI & 17,47 & Long A & Jap & ITA & 143 & 7,72 & 2,83 & 2,73 & 19 & $\mathrm{~T}$ & A & G & $\mathrm{T}$ & A & C & \\
\hline OSCAR & 17,56 & Long A & Jap & PRT & 166 & 7,12 & 2,40 & 2,97 & 18 & $\mathrm{~T}$ & A & G & $\mathrm{T}$ & A & C & \\
\hline PERLA & 17,67 & Round & Jap & ITA & 150 & 5,14 & 2,88 & 1,78 & 18 & $\mathrm{~T}$ & A & G & $\mathrm{T}$ & A & C & \\
\hline ROXANI & 17,72 & Long A & Jap & GRC & 162 & 7,01 & 2,96 & 2,37 & 18 & $\mathrm{~T}$ & A & G & $\mathrm{T}$ & A & C & \\
\hline SELENIO & 17,75 & Round & Jap & ITA & 155 & 5,01 & 2,90 & 1,73 & 17 & $\mathrm{~T}$ & A & G & $\mathrm{T}$ & A & C & \\
\hline ARBORIO & 17,79 & Long A & Jap & ITA & 149 & 7,21 & 3,50 & 2,06 & 18 & $\mathrm{~T}$ & A & G & $\mathrm{T}$ & A & C & \\
\hline GRITNA & 17,83 & Long A & Jap & ITA & 140 & 7,21 & 2,94 & 2,45 & 18 & $\mathrm{~T}$ & A & $\mathrm{G}$ & $\mathrm{T}$ & A & C & $\frac{6}{10}$ \\
\hline S 101 & 17,85 & Medium & Jap & USA & 153 & 5,28 & 2,93 & 1,80 & 18 & $\mathrm{~T}$ & A & G & $\mathrm{T}$ & A & C & \\
\hline
\end{tabular}


Table 1 Germplasm collection of 127 rice accessions (Continued)

\begin{tabular}{|c|c|c|c|c|c|c|c|c|c|c|c|c|c|c|c|}
\hline AUGUSTO & 18,06 & Long A & Jap & ITA & 143 & 6,86 & 2,69 & 2,55 & 18 & $\mathrm{~T}$ & $A$ & $G$ & $\mathrm{~T}$ & A & C \\
\hline ARIETE & 18,08 & Long A & Jap & ITA & 145 & 6,81 & 2,64 & 2,58 & 18 & $\mathrm{~T}$ & $A$ & G & $\mathrm{T}$ & A & C \\
\hline GIZA 177 & 18,10 & Medium & Jap & EGY & 158 & 5,36 & 2,90 & 1,85 & 17 & $\mathrm{~T}$ & A & G & $T$ & A & C \\
\hline SAVIO & 18,11 & Long A & Jap & ITA & 151 & 6,57 & 2,60 & 2,53 & 18 & $\mathrm{~T}$ & $A$ & G & $T$ & A & C \\
\hline LOTO & 18,20 & Long A & Jap & ITA & 139 & 6,51 & 2,95 & 2,21 & 18 & $\mathrm{~T}$ & A & G & $T$ & A & C \\
\hline KORAL & 18,27 & Long A & Jap & ITA & 151 & 6,89 & 2,74 & 2,51 & 18 & $\mathrm{~T}$ & A & G & $T$ & A & C \\
\hline Т 757 & 18,30 & Long A & Jap & IND & 162 & 6,22 & 3,20 & 1,94 & 18 & $\mathrm{~T}$ & $A$ & G & $T$ & A & C \\
\hline SHSS 53 & 18,44 & Long A & Jap & ESP & 158 & 6,63 & 2,96 & 2,24 & 18 & $\mathrm{~T}$ & $A$ & G & $\mathrm{T}$ & A & C \\
\hline SCUDO & 18,49 & Long B & Jap & ITA & 163 & 7,53 & 2,29 & 3,29 & 18 & $\mathrm{~T}$ & A & G & $\mathrm{T}$ & A & C \\
\hline CHACARERO & 18,55 & Long A & Jap & USA & 164 & 7,84 & 3,00 & 2,61 & 18 & $\mathrm{~T}$ & A & G & $T$ & A & C \\
\hline M 202 & 18,55 & Medium & Jap & USA & 152 & 6,00 & 2,82 & 2,13 & 18 & $\mathrm{~T}$ & A & G & $\mathrm{T}$ & A & C \\
\hline MELAS & 18,61 & Long B & Jap & GRC & 154 & 7,04 & 2,34 & 3,01 & 18 & $\mathrm{~T}$ & A & G & $\mathrm{T}$ & A & C \\
\hline GUITA & 18,62 & Medium & Jap & PHL & 162 & 5,90 & 2,98 & 1,98 & 18 & $\mathrm{~T}$ & A & G & $T$ & A & C \\
\hline IR56381-139-2-2 & 18,65 & Long A & Jap & ARG & 160 & 6,34 & 3,05 & 2,08 & 18 & $\mathrm{~T}$ & $A$ & G & $T$ & A & C \\
\hline $\begin{array}{l}\text { LA PLATA } \\
\text { GUANEJAN }\end{array}$ & 18,65 & Long A & Jap & $\mathrm{PHL}$ & 161 & 7,53 & 3,01 & 2,50 & 18 & $\mathrm{~T}$ & A & G & $\mathrm{T}$ & A & C \\
\hline MARATELLI & 18,66 & Medium & Jap & ITA & 145 & 5,61 & 3,13 & 1,79 & 18 & $\mathrm{~T}$ & $A$ & G & $\mathrm{T}$ & A & C \\
\hline KULON & 18,76 & Long A & Jap & RUS & 147 & 6,55 & 2,91 & 2,25 & 18 & $\mathrm{~T}$ & A & G & $\mathrm{T}$ & A & C \\
\hline $\begin{array}{l}\text { ESTRELA } \\
\text { IRRADIADO }\end{array}$ & 18,80 & Long A & Jap & PRT & 154 & 6,50 & 2,93 & 2,22 & 18 & $\mathrm{~T}$ & A & G & $\mathrm{T}$ & A & C \\
\hline BENGAL & 18,81 & Long A & Jap & USA & 163 & 6,11 & 2,74 & 2,23 & 18 & $\mathrm{~T}$ & A & G & $\mathrm{T}$ & A & C \\
\hline CENTAURO & 18,86 & Round & Jap & ITA & 155 & 5,60 & 3,36 & 1,67 & 18 & $\mathrm{~T}$ & $A$ & G & $T$ & A & C \\
\hline LUXOR & 18,88 & Long A & Jap & ITA & 158 & 6,48 & 3,02 & 2,15 & 18 & $\mathrm{~T}$ & A & G & $\mathrm{T}$ & A & C \\
\hline CIGALON & 18,92 & Medium & Jap & FRA & 140 & 5,38 & 3,14 & 1,71 & 18 & $\mathrm{~T}$ & $A$ & G & $\mathrm{T}$ & A & C \\
\hline ERCOLE & 19,00 & Long A & Jap & ITA & 157 & 7,02 & 2,88 & 2,44 & 19 & $\mathrm{~T}$ & A & G & $\mathrm{T}$ & A & C \\
\hline THAIPERLA & 19,09 & Round & Jap & USA & 161 & 5,55 & 3,25 & 1,71 & 18 & $\mathrm{~T}$ & A & G & $\mathrm{T}$ & A & C \\
\hline S. ANDREA & 19,14 & Long A & Jap & ITA & 157 & 6,92 & 3,29 & 2,10 & 18 & $\mathrm{~T}$ & $A$ & G & $T$ & A & C \\
\hline SIS R215 & 19,17 & Long A & Jap & ITA & 153 & 7,30 & 2,57 & 2,84 & 18 & $\mathrm{~T}$ & A & G & $T$ & A & C \\
\hline COLINA & 19,25 & Round & Jap & ESP & 161 & 5,18 & 3,06 & 1,69 & 18 & $\mathrm{~T}$ & A & G & $\mathrm{T}$ & A & C \\
\hline FLIPPER & 19,28 & Medium & Jap & ITA & 152 & 5,99 & 3,01 & 1,99 & 18 & $\mathrm{~T}$ & A & G & $T$ & A & C \\
\hline ITALPATNA 48 & 19,34 & Long A & Jap & ITA & 151 & 7,12 & 2,60 & 2,74 & 17 & $\mathrm{~T}$ & A & G & $\mathrm{T}$ & A & C \\
\hline M 204 & 19,42 & Long A & Jap & USA & 156 & 6,19 & 2,85 & 2,17 & 18 & $\mathrm{~T}$ & A & G & $\mathrm{T}$ & A & C \\
\hline SAKHA 102 & 19,43 & Medium & Jap & EGY & 166 & 5,59 & 2,90 & 1,93 & 17 & $\mathrm{~T}$ & A & G & $\mathrm{T}$ & A & C \\
\hline CENTURY PATNA & 19,52 & Long A & Jap & USA & 157 & 8,00 & 3,15 & 2,54 & 18 & $\mathrm{~T}$ & A & G & $\mathrm{T}$ & A & C \\
\hline
\end{tabular}


Table 1 Germplasm collection of 127 rice accessions (Continued)

\begin{tabular}{|c|c|c|c|c|c|c|c|c|c|c|c|c|c|c|c|}
\hline SALVO & 19,67 & Long B & Jap & ITA & 154 & 7,36 & 2,12 & 3,47 & 18 & $\mathrm{~T}$ & $A$ & G & $\mathrm{T}$ & $A$ & C \\
\hline PECOS & 19,77 & Medium & Jap & USA & 161 & 5,62 & 2,81 & 2,00 & 18 & $\mathrm{~T}$ & A & G & $T$ & A & C \\
\hline 89 AXHVA-6 & 19,97 & Round & Jap & ARG & 166 & 5,69 & 3,13 & 1,82 & 18 & $\mathrm{~T}$ & A & G & $\mathrm{T}$ & A & C \\
\hline Upla 104 & 19,97 & Long A & Jap & ARG & 168 & 7,95 & 3,02 & 2,63 & 18 & $\mathrm{~T}$ & A & G & $\mathrm{T}$ & A & C \\
\hline CT 23 & 20,03 & Long B & Jap & $\mathrm{COL}$ & 157 & 7,93 & 2,45 & 3,24 & 18 & $\mathrm{~T}$ & A & G & $\mathrm{T}$ & A & C \\
\hline FIDJI & 20,05 & Long B & Jap & $\mathrm{PHL}$ & 163 & 7,88 & 2,23 & 3,53 & 18 & $\mathrm{~T}$ & A & G & $\mathrm{T}$ & A & C \\
\hline S $102-2$ & 20,09 & Long A & Jap & USA & 164 & 5,62 & 3,23 & 1,74 & 18 & $\mathrm{~T}$ & A & G & $\mathrm{T}$ & A & C \\
\hline MAIORAL & 20,18 & Long A & Jap & PRT & 154 & 7,96 & 2,83 & 2,81 & 18 & $\mathrm{~T}$ & $A$ & G & $\mathrm{T}$ & A & C \\
\hline BELOZEM & 20,21 & Medium & Jap & $B G R$ & 157 & 6,04 & 3,25 & 1,86 & 18 & $\mathrm{~T}$ & A & G & $\mathrm{T}$ & A & C \\
\hline SUWEON 280 & 20,34 & Long A & Jap & $\mathrm{KOR}$ & - & 6,00 & 2,64 & 2,72 & 18 & $\mathrm{~T}$ & A & G & $\mathrm{T}$ & A & C \\
\hline GRAAL & 20,40 & Long B & Jap & FRA & 149 & 7,63 & 2,23 & 3,42 & 18 & $\mathrm{~T}$ & A & G & $\mathrm{T}$ & A & C \\
\hline CAPATAZ & 20,53 & Long A & Jap & ESP & 157 & 6,31 & 3,23 & 1,95 & 18 & $\mathrm{~T}$ & A & G & $T$ & A & C \\
\hline UPLA 80 & 20,54 & Long B & Jap & ARG & 142 & 7,81 & 2,12 & 3,68 & 18 & $\mathrm{~T}$ & A & G & $\mathrm{T}$ & A & C \\
\hline ROTUNDUS & 20,65 & Long A & Jap & HUN & 139 & 8,75 & 3,46 & 2,53 & 17 & G & $C$ & $\mathrm{~T}$ & $T$ & A & C \\
\hline $\begin{array}{l}\text { ITALPATNA X } \\
\text { MILYANG }\end{array}$ & 20,96 & Long A & Jap & PRT & 150 & 6,69 & 2,72 & 2,46 & 18 & $\mathrm{~T}$ & A & G & $\mathrm{T}$ & A & C \\
\hline UPLA 91 & 21,21 & Long A & Jap & ARG & 140 & 7,11 & 2,43 & 2,93 & 18 & $\mathrm{~T}$ & A & G & $\mathrm{T}$ & A & C \\
\hline ANTONI & 21,27 & Long A & Jap & $B G R$ & 131 & 6,49 & 2,91 & 2,23 & 17 & G & $C$ & $\mathrm{~T}$ & $\mathrm{~T}$ & A & C \\
\hline GOOLARAH & 21,30 & Long B & Jap & AUS & 174 & 7,62 & 2,16 & 3,53 & 19 & $\mathrm{~T}$ & A & G & $\mathrm{T}$ & A & C \\
\hline UPLA 68 & 21,56 & Long B & Jap & ARG & 149 & 8,02 & 2,29 & 3,50 & 18 & $\mathrm{~T}$ & A & G & $\mathrm{T}$ & A & C \\
\hline JEFFERSON & 22,21 & Long A & Jap & USA & 157 & 6,79 & 2,44 & 2,78 & 20 & G & $C$ & G & $\mathrm{T}$ & A & C \\
\hline SAFARI & 22,37 & Long A & Jap & PRT & 149 & 6,60 & 2,72 & 2,43 & 17 & G & $C$ & $\mathrm{~T}$ & $\mathrm{~T}$ & A & C \\
\hline A 301 & 22,44 & Long B & Jap & USA & 163 & 7,78 & 2,26 & 3,44 & 20 & G & $C$ & G & $\mathrm{T}$ & A & C \\
\hline OTA & 22,55 & Long A & Jap & PRT & 161 & 6,15 & 2,90 & 2,12 & 13 & G & $C$ & $\mathrm{~T}$ & $\mathrm{~T}$ & A & C \\
\hline UPLA 64 & 22,57 & Long B & Jap & ARG & 148 & 7,87 & 2,16 & 3,64 & 17 & G & $C$ & $\mathrm{~T}$ & $\mathrm{~T}$ & A & C \\
\hline LORD & 22,60 & Long A & Jap & ITA & 144 & 6,93 & 2,60 & 2,67 & 20 & G & $C$ & G & $\mathrm{T}$ & A & C \\
\hline SANDORA & 22,75 & Long A & Jap & HUN & 135 & 7,39 & 2,62 & 2,82 & 17 & G & $C$ & $\mathrm{~T}$ & $T$ & A & C \\
\hline DELLROSE & 22,83 & Long A & Jap & USA & 163 & 6,58 & 2,27 & 2,90 & 20 & G & $C$ & G & $\mathrm{T}$ & A & C \\
\hline BOMBA & 22,84 & Medium & Jap & ESP & 159 & 5,45 & 2,99 & 1,82 & 21 & G & $C$ & $\mathrm{~T}$ & $\mathrm{~T}$ & A & C \\
\hline N 3 & 22,86 & Long A & Jap & ESP & 167 & 6,64 & 2,58 & 2,57 & 20 & G & $C$ & G & $\mathrm{T}$ & A & C \\
\hline KARNAK & 22,90 & Long A & Jap & ITA & 156 & 7,45 & 3,43 & 2,17 & 17 & G & $C$ & $\mathrm{~T}$ & $\mathrm{~T}$ & A & C \\
\hline $\begin{array}{l}\text { GIGANTE } \\
\text { VERCELLI }\end{array}$ & 23,05 & Long A & Jap & ITA & 149 & 6,95 & 3,32 & 2,09 & 17 & G & $C$ & $\mathrm{~T}$ & $\mathrm{~T}$ & A & C \\
\hline MARTA & 23,06 & Long B & Jap & ITA & 183 & 7,40 & 2,29 & 3,23 & 20 & G & $C$ & G & $\mathrm{T}$ & A & C \\
\hline
\end{tabular}


Table 1 Germplasm collection of 127 rice accessions (Continued)

\begin{tabular}{|c|c|c|c|c|c|c|c|c|c|c|c|c|c|c|c|}
\hline COCODRIE & 23,10 & Long B & Jap & USA & 155 & 7,01 & 2,10 & 3,34 & 20 & G & $A$ & G & $\mathrm{T}$ & $A$ & C \\
\hline DREW & 23,26 & Long B & Jap & USA & 160 & 6,81 & 2,16 & 3,15 & 14 & G & $C$ & $\mathrm{~T}$ & $\mathrm{~T}$ & A & C \\
\hline DELLMONT & 23,33 & Long B & Jap & USA & 160 & 6,96 & 2,27 & 3,07 & 20 & G & $C$ & G & $\mathrm{T}$ & A & C \\
\hline IR 5549-1-2 & 23,47 & Long B & Jap & $\mathrm{PHL}$ & 173 & 6,98 & 2,25 & 3,10 & 20 & G & $C$ & G & $\mathrm{T}$ & A & C \\
\hline L 205 & 23,57 & Long B & Jap & USA & 156 & 7,47 & 2,21 & 3,38 & 10 & G & A & $T$ & $C$ & G & $\mathrm{T}$ \\
\hline BLUEBONNET & 23,60 & Long B & Jap & USA & - & 8,16 & 2,66 & 3,07 & 20 & G & $C$ & G & $\mathrm{T}$ & A & C \\
\hline TEJO & 23,63 & Long A & Jap & ITA & 156 & 6,71 & 2,63 & 2,55 & 20 & G & $C$ & G & $\mathrm{T}$ & A & C \\
\hline GOLFO & 23,75 & Long A & Jap & ITA & 181 & 7,39 & 2,47 & 2,99 & 20 & G & $C$ & G & $\mathrm{T}$ & A & C \\
\hline LAGRUE & 23,76 & Long A & Jap & USA & 160 & 6,50 & 2,20 & 2,95 & 20 & G & $C$ & G & $\mathrm{T}$ & A & C \\
\hline DORADO & 23,79 & Long B & Jap & GRC & 164 & 7,29 & 2,23 & 3,27 & 14 & G & $C$ & $\mathrm{~T}$ & $\mathrm{~T}$ & A & C \\
\hline PLUS & 23,84 & Long B & Jap & ITA & 159 & 7,02 & 2,26 & 3,11 & 14 & G & $C$ & $\mathrm{~T}$ & $\mathrm{~T}$ & A & C \\
\hline MAYBELLE & 23,87 & Long B & Jap & USA & 155 & 6,65 & 2,19 & 3,04 & 20 & G & $C$ & G & $\mathrm{T}$ & A & C \\
\hline L 204 & 23,90 & Long B & Jap & USA & 153 & 7,81 & 2,37 & 3,30 & 20 & G & $C$ & G & $\mathrm{T}$ & A & C \\
\hline GIADA & 23,93 & Long B & Jap & ITA & 151 & 7,45 & 2,08 & 3,58 & 17 & G & $C$ & $\mathrm{~T}$ & $\mathrm{~T}$ & A & C \\
\hline BOND & 23,96 & Long B & Jap & USA & 157 & 7,08 & 2,24 & 3,16 & 20 & G & $C$ & G & $\mathrm{T}$ & A & C \\
\hline MERLE' & 24,18 & Long B & Jap & FRA & 151 & 7,08 & 2,23 & 3,17 & 9 & G & A & $T$ & $C$ & G & $\mathrm{T}$ \\
\hline REXMONT & 24,19 & Long B & Jap & USA & 161 & 6,72 & 2,19 & 3,07 & 10 & G & A & $\mathrm{T}$ & $C$ & G & $\mathrm{T}$ \\
\hline LACASSINE & 24,21 & Long B & Jap & USA & 162 & 7,03 & 2,21 & 3,18 & 20 & G & $C$ & G & $\mathrm{T}$ & A & C \\
\hline ALAN & 24,30 & Long B & Jap & USA & 154 & 6,86 & 2,03 & 3,38 & 14 & G & A & $T$ & $\mathrm{~T}$ & A & C \\
\hline DIXIEBELLE & 24,35 & Long A & Jap & USA & 167 & 6,28 & 2,21 & 2,84 & 11 & G & A & $\mathrm{T}$ & $C$ & G & $\mathrm{T}$ \\
\hline ARIANA & 24,42 & Long B & Jap & $\mathrm{ROM}$ & 168 & 7,34 & 2,41 & 3,04 & 20 & G & $C$ & G & $\mathrm{T}$ & A & C \\
\hline CNA 4081 & 24,44 & Long B & Ind & BRA & 149 & 6,64 & 2,19 & 3,03 & 9 & G & A & $T$ & $C$ & G & $\mathrm{T}$ \\
\hline A 201 & 24,50 & Long B & Jap & USA & 165 & 7,83 & 2,11 & 3,71 & 20 & G & A & G & $\mathrm{T}$ & A & C \\
\hline GLADIO & 24,73 & Long B & Jap & ITA & 129 & 7,28 & 2,19 & 3,32 & 20 & G & A & G & $\mathrm{T}$ & A & C \\
\hline THAIBONNET & 24,83 & Long B & Jap & USA & 159 & 7,81 & 2,37 & 3,30 & 20 & G & A & G & $\mathrm{T}$ & A & C \\
\hline ORIONE & 24,85 & Long A & Jap & ITA & 173 & 6,90 & 2,65 & 2,60 & 9 & G & A & $\mathrm{T}$ & $C$ & G & $\mathrm{T}$ \\
\hline IR 47686-9-4-1 & 25,03 & Long B & Jap & $\mathrm{PHL}$ & 172 & 7,14 & 2,19 & 3,26 & 20 & G & A & G & $\mathrm{T}$ & A & C \\
\hline FRAGRANCE & 25,16 & Long B & Jap & ITA & 154 & 7,74 & 2,42 & 3,20 & 20 & G & A & G & $\mathrm{T}$ & A & C \\
\hline L.202 & 25,21 & Long B & Jap & USA & 155 & 7,52 & 2,18 & 3,45 & 20 & G & A & G & $\mathrm{T}$ & A & C \\
\hline ZHEN SHANG 47 & 25,40 & Long A & Ind & $\mathrm{CHN}$ & 152 & 6,10 & 2,92 & 2,09 & 11 & G & A & G & $C$ & G & $\mathrm{T}$ \\
\hline ARROYOGRANDE & 25,46 & Long B & Jap & ESP & 159 & 7,74 & 2,20 & 3,52 & 20 & G & A & G & $\mathrm{T}$ & A & C \\
\hline ALINANO C & 26,03 & Long A & Jap & FRA & 151 & 7,38 & 2,57 & 2,87 & 11 & G & A & G & $C$ & G & $\mathrm{T}$ \\
\hline
\end{tabular}




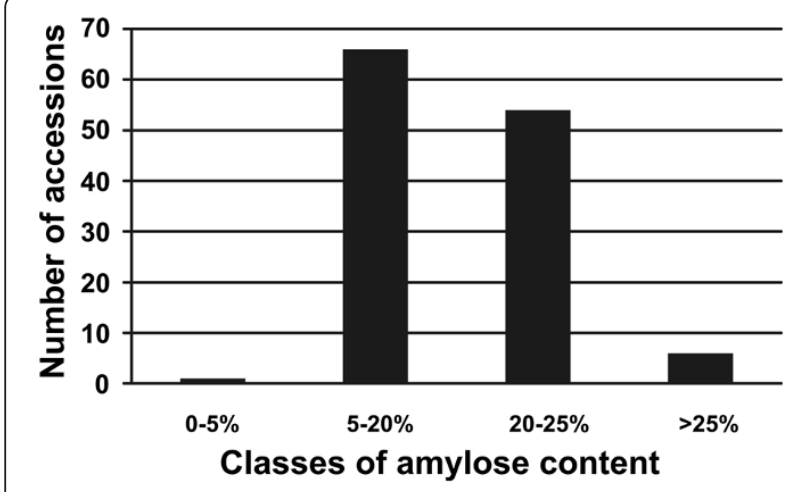

Figure 1 Frequency distribution of amylose content classes in the analyzed germplasm collection: Waxy (0-5\%), low amylose $(5-20 \%)$, intermediate $(20-25 \%)$ and high amylose $(>25 \%)$.

not observe mutations in exon 10 thus excluding the presence of SNPs in this exon.

Combining the results obtained for SNPs in intron 1 and exon 6 , three allelic patterns were identified: GA, TA and GC (Table 2), the first letter indicating the G/T polymorphism in intron 1 while the second the A/C SNP in exon 6. Similarly to the behaviour observed for intron 1, most of the accessions from waxy to $22 \%$ AAC (with two exceptions) carried the TA haplotype (Table 1), GC pattern was present in accessions with 22-24\% AAC (with two exceptions) and GA in accessions with AAC >24\% (with three exceptions). Statistical analyses showed that the A/C SNP in exon 6 alone explained only the $30.9 \%$ of the variation in AAC, but altogether the two SNPs explained the $79.5 \%$. Adding the RM190 microsatellite to the analysis, we did not find a significantly higher explanation of the AAC variation: $79.6 \%$ vs. $79.5 \%$ (Table 3 ).

Table 2 Alleles identified for the RM190 microsatellite in the germplasm collection

\begin{tabular}{lllll}
\hline $\begin{array}{l}\text { CT-repeats } \\
\text { (RM190) }\end{array}$ & $\begin{array}{l}\text { Allelic pattern } \\
\text { SNPs intron } \\
\mathbf{1}_{\text {and exon } \mathbf{6}^{\mathbf{a}}}\end{array}$ & $\begin{array}{l}\text { Number } \\
\text { of cvs. }\end{array}$ & $\begin{array}{l}\text { AAC } \\
\text { range (\%) }\end{array}$ & $\begin{array}{l}\text { AAC } \\
\text { average (\%) }\end{array}$ \\
\hline 9 & GA & 3 & $24.18-24.85$ & 24.49 \\
10 & GA & 2 & $23.57-24.19$ & 23.88 \\
11 & GA & 3 & $24.35-26.03$ & 25.26 \\
13 & GC & 1 & 22.55 & \\
14 & GA & 4 & $23.06-24.30$ & 23.80 \\
17 & TA & 6 & $16.42-23.27$ & 19.05 \\
17 & GC & 8 & $20.65-23.93$ & 22.44 \\
18 & TA & 67 & $14.92-21.56$ & 18.37 \\
19 & TA & 6 & $15.55-21.30$ & 17.78 \\
20 & GA & 7 & $23.10-25.46$ & 24.71 \\
20 & GC & 18 & $22.21-25.03$ & 23.50 \\
21 & GC & 1 & 22.84 & \\
\hline Th & G & 6 & & \\
\hline
\end{tabular}

${ }^{a}$ The two bases indicate the SNP alleles in intron 1 and in exon 6, respectively.
Table 3 Percentage of variation for Apparent Amylose Content (AAC) explained by the tested GBSSI molecular markers in the germplasm collection

\begin{tabular}{|c|c|c|}
\hline Polymorphisms & $\begin{array}{l}\text { Explained AAC } \\
\text { variation (\%) }\end{array}$ & $p$ values \\
\hline CTn (RM190) & 74.9 & 0.000 \\
\hline T/G intron1 & 77.5 & 0.000 \\
\hline $\mathrm{A} / \mathrm{C}$ exon 6 & 30.9 & 0.000 \\
\hline \multirow[t]{2}{*}{ CTn/intron1 } & 77.7 & $0.260 \mathrm{CTn}$ \\
\hline & & 0.000 intron 1 \\
\hline \multirow[t]{2}{*}{ CTn/exon6 } & 40.3 & $0.000 \mathrm{CTn}$ \\
\hline & & 0.000 exon6 \\
\hline \multirow[t]{2}{*}{ Intron 1/exon6 } & 79.5 & 0.000 intron 1 \\
\hline & & 0.001 exon6 \\
\hline \multirow[t]{3}{*}{ CTn/intron1/exon6 } & 79.6 & $0.594 \mathrm{CTn}$ \\
\hline & & 0.000 intron 1 \\
\hline & & 0.001 exon6 \\
\hline \multirow[t]{3}{*}{ Intron1/exon6/-1,514 } & 80.1 & 0.000 intron 1 \\
\hline & & 0.001 exon6 \\
\hline & & $0.072-1,514$ \\
\hline \multirow[t]{4}{*}{ CTn/intron1/exon6/-1,514 } & 80.3 & $0.242 C T n$ \\
\hline & & 0.000 intron 1 \\
\hline & & 0.035 exon6 \\
\hline & & $0.038-1,514$ \\
\hline \multirow[t]{4}{*}{ intron1/exon6/-1,514/exon9 } & 80.1 & 0.000 intron 1 \\
\hline & & 0.001 exon6 \\
\hline & & $0.073-1,514$ \\
\hline & & 0.965 exon9 \\
\hline \multirow[t]{5}{*}{ CTn/intron1/exon6/-1,514/exon9 } & 80.3 & $0.216 C T n$ \\
\hline & & 0.000 intron 1 \\
\hline & & 0.038 exon6 \\
\hline & & $0.035-1,514$ \\
\hline & & 0.675 exon9 \\
\hline
\end{tabular}

The allelic variation at the RM190 locus together with intron 1/exon 6 SNPs data allowed the identification of allelic patterns associated to different AAC classes. In particular, even considering that no association of $\mathrm{CT}_{17}$ with a specific AAC group could be identified, all the accessions with haplotype $\mathrm{CT}_{17}, \mathrm{G}$ in intron 1 and $\mathrm{C}$ in exon 6 shared an AAC level ranging from 22 to 23\%, with the exception of Giada (23.93\%). Similarly, the $\mathrm{CT}_{14}$ allele associated with the CG and AG allelic patterns shared similar AAC (ranging from 23.2 to 23.8\%). Among the $\mathrm{CT}_{20}$ group, the most frequent allelic pattern for high AAC class was CG, which identifies an AAC range from 22 to $24 \%$, while in combination with AG frequently identified accessions with more than $25 \%$ 
AAC. $\mathrm{CT}_{9}, \mathrm{CT}_{10}$ and $\mathrm{CT}_{11}$ associated to the allelic pattern AG were typical of genotypes with more than $24 \%$ of AAC.

Despite the fact that the level of variation in AAC explained by our results is in agreement to the one recently evaluated in an Italian rice collection (Caffagni et al. 2013), it is consistently lower than previously observed. As examples, Ayres et al. (1997) using the combination of RM190 and the G/T in intron1 explained the 85.9\% of the variation in AAC; Dobo and co-workers (2010) could explain the $93.8 \%$ of variation in AAC with RM190, the SNP in intron 1, the SNP in exon 6 and the SNP in exon 10 which was not present in our collection. Owing these results, it was realized that additional molecular markers could be needed to increase predictability of the different AAC classes within our germplasm panel.

\section{Allele mining of the GBSSI gene}

To mine the genetic variation at the level of the GBSSI locus within our germplasm collection, the gene as well as $1 \mathrm{kbp}$ of the upstream putative regulatory region were sequenced in twenty-one genotypes representing all the AAC classes identified: Calmochi 101 for the waxy type; Prever, Yrl 196, Delta, Lomellino and Campino for 1416\% AAC; Yrm 6-2, Timich 108, Augusto, Loto and Sant'Andrea for 16-19\% AAC; Upla 91, Antoni, Gigante Vercelli, A201 and Gladio for 21-25\% AAC; and Fragrance, L 202, Zhen Shang 47, Arroyogrande and Alinano $\mathrm{C}$ for $>25 \%$ AAC (Table 1). The twenty-one GBSSI sequences were compared by multiple alignments considering the Nipponbare sequence as the reference. Sequence comparisons showed an overall high level of similarity (Figure 2), indicating that the coding sequence was conserved in most of the genotypes with some exceptions and led to the identification of 32 SNPs (Table 4). The waxy cv. Calmochi 101 carried the wx allele with the $23 \mathrm{bp}$ duplication in exon 2, as described before. Antoni and Gigante Vercelli accessions showed the non-conservative A/C SNP in exon 6, previously identified and causing a Serine/Tyrosine substitution in the GBSSI protein (Larking and Park, 2003). Additionally, these genotypes contained two common SNPs: one in the putative regulatory region (position $-1,514$ ) and one in the first intron (position -399). Gigante Vercelli also showed a SNP at $-2,174$ bp upstream the ATG (Figure 2; Table 4). Zhen Shang 47 and Alinano $C$ carried a C/T SNP at position $+1,801$ in exon 9 which results in the substitution of Pro415 to Ser in the GBSS protein. Also for Zhen Shang 47 and Alinano C, SNP mutations were identified in the non-coding sequences: 2 in the promoter region, 11 in intron 1,1 in intron 6, 9 in intron 10 and 2 in intron 12 (Figure 2; Table 4). Most of the SNPs identified in the present work are not classified in the group of SNPs computationally characterized by Kharabian (2010) and present in OryzaSNP (http:// oryzasnp.plantbiology.msu.edu/cgi-bin/gbrowse/osa_snp_ tigr/) and dbSNP (http://www.ncbi.nlm.nih.gov/sites/entrez? $\mathrm{db}=$ snp\&TAbCmd=Limits) databases. Additionally to these SNPs polymorphisms, a 4 bp tandem repeat that was

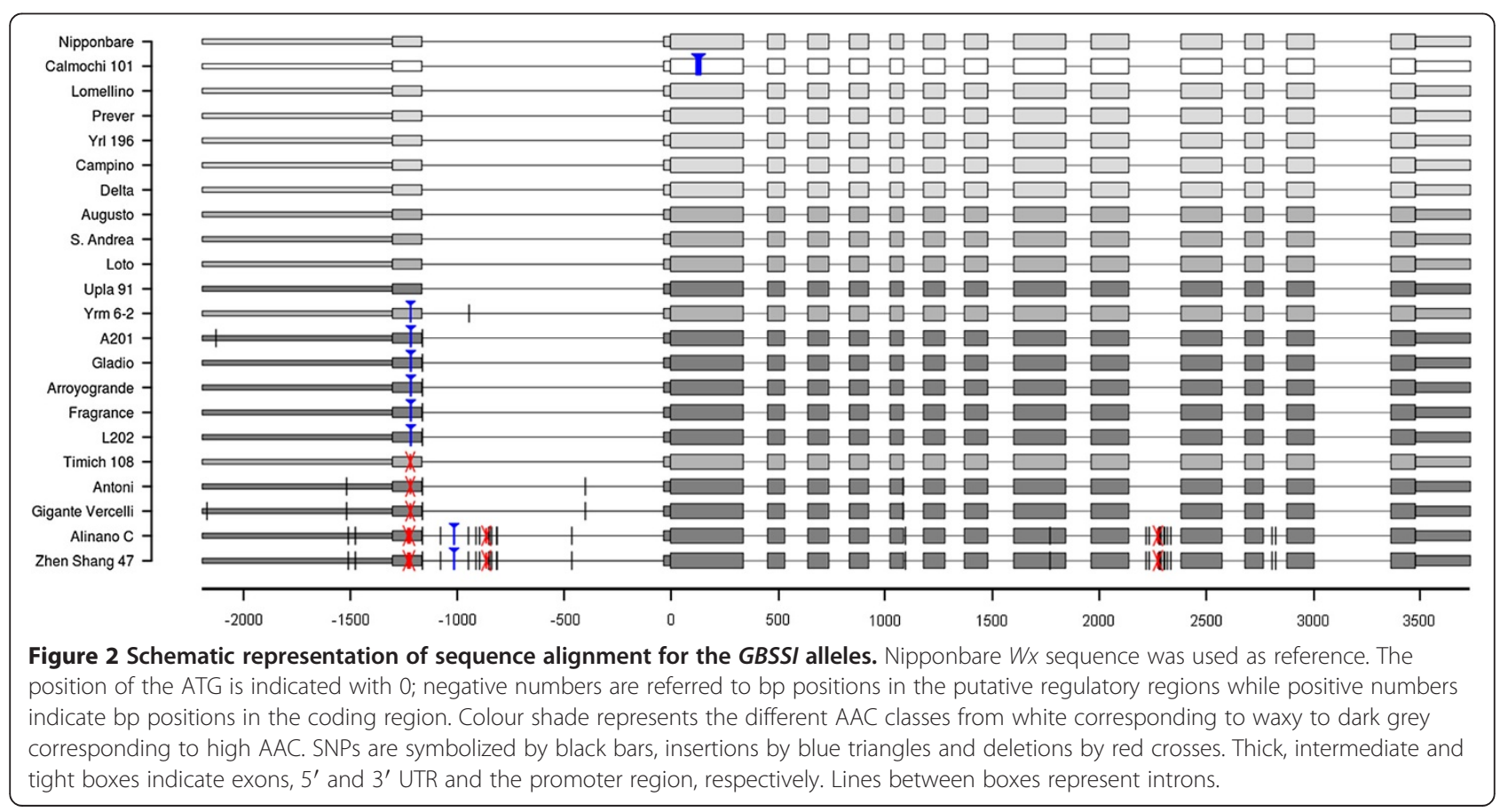


Table 4 SNPs identified from re-sequencing of the Waxy gene and the putative regulatory region

\begin{tabular}{|c|c|c|c|c|}
\hline Genotype & AAC (\%) & SNP & Position & OryzaSNP ID \\
\hline Yrm 6-2 & 16.40 & $\mathrm{G} / \mathrm{A}$ & -942 & $n c^{a}$ \\
\hline Gladio & 24.73 & $\mathrm{~T} / \mathrm{G}$ & -1164 & $\mathrm{nc}$ \\
\hline Arroyogrande & 25.46 & $\mathrm{~T} / \mathrm{G}$ & -1164 & $\mathrm{nc}$ \\
\hline Fragrance & 25.16 & $\mathrm{~T} / \mathrm{G}$ & -1164 & $\mathrm{nc}$ \\
\hline L202 & 25.21 & $\mathrm{~T} / \mathrm{G}$ & -1164 & $\mathrm{nc}$ \\
\hline \multirow[t]{2}{*}{ A201 } & 24.50 & $\mathrm{~A} / \mathrm{G}$ & -2132 & $\mathrm{nc}$ \\
\hline & & $\mathrm{T} / \mathrm{G}$ & -1164 & $\mathrm{nc}$ \\
\hline \multirow[t]{4}{*}{ Antoni } & 21.27 & $\mathrm{G} / \mathrm{T}$ & -1514 & $\mathrm{nc}$ \\
\hline & & $T / G$ & -1164 & $\mathrm{nc}$ \\
\hline & & $\mathrm{G} / \mathrm{T}$ & -399 & $\mathrm{nc}$ \\
\hline & & $\mathrm{A} / \mathrm{C}$ & +1083 & TBGI270316 \\
\hline \multirow[t]{5}{*}{ Gigante Vercelli } & 23.05 & T/A & -2174 & $\mathrm{nc}$ \\
\hline & & $\mathrm{G} / \mathrm{T}$ & -1514 & $\mathrm{nc}$ \\
\hline & & $\mathrm{T} / \mathrm{G}$ & -1164 & $\mathrm{nc}$ \\
\hline & & $\mathrm{G} / \mathrm{T}$ & -399 & nc \\
\hline & & $\mathrm{A} / \mathrm{C}$ & +1083 & TBGI270316 \\
\hline \multirow[t]{26}{*}{ Zhen Shang/Alinano C } & $25.40 / 26.03$ & $A / G$ & -1522 & $\mathrm{nc}$ \\
\hline & & $\mathrm{C} / \mathrm{T}$ & -1481 & $\mathrm{nc}$ \\
\hline & & $\mathrm{T} / \mathrm{G}$ & -1164 & $\mathrm{nc}$ \\
\hline & & $\mathrm{G} / \mathrm{A}$ & -1079 & $\mathrm{nc}$ \\
\hline & & $\mathrm{A} / \mathrm{G}$ & -945 & $\mathrm{nc}$ \\
\hline & & $\mathrm{C} / \mathrm{T}$ & -918 & $\mathrm{nc}$ \\
\hline & & $\mathrm{A} / \mathrm{G}$ & -901 & $\mathrm{nc}$ \\
\hline & & $C / T$ & -850 & $\mathrm{nc}$ \\
\hline & & $\mathrm{A} / \mathrm{C}$ & -847 & $\mathrm{nc}$ \\
\hline & & $T / G$ & -837 & $\mathrm{nc}$ \\
\hline & & $\mathrm{C} / \mathrm{T}$ & -813 & $\mathrm{nc}$ \\
\hline & & $\mathrm{T} / \mathrm{C}$ & -811 & $\mathrm{nc}$ \\
\hline & & $\mathrm{T} / \mathrm{C}$ & -461 & $\mathrm{nc}$ \\
\hline & & $C / T$ & +1094 & TBGI270317 \\
\hline & & $C / T$ & $+1,801$ & $\mathrm{nc}$ \\
\hline & & $\mathrm{G} / \mathrm{A}$ & +2218 & $\mathrm{nc}$ \\
\hline & & $\mathrm{G} / \mathrm{A}$ & +2232 & $\mathrm{nc}$ \\
\hline & & $\mathrm{A} / \mathrm{G}$ & +2279 & $\mathrm{nc}$ \\
\hline & & $\mathrm{G} / \mathrm{A}$ & +2282 & $\mathrm{nc}$ \\
\hline & & $\mathrm{T} / \mathrm{C}$ & +2288 & $\mathrm{nc}$ \\
\hline & & $\mathrm{G} / \mathrm{A}$ & +2301 & $\mathrm{nc}$ \\
\hline & & $\mathrm{T} / \mathrm{C}$ & +2305 & $\mathrm{nc}$ \\
\hline & & $C / G$ & +2317 & $\mathrm{nc}$ \\
\hline & & $\mathrm{A} / \mathrm{G}$ & +2333 & $\mathrm{nc}$ \\
\hline & & $C / T$ & +2806 & $\mathrm{nc}$ \\
\hline & & $\mathrm{G} / \mathrm{A}$ & +2823 & TBGI270324 \\
\hline
\end{tabular}

For each polymorphism, the rice accession where it was identified, the nucleotide mutation, the position with respect to the ATG and the ID in the OryzaSNP database are indicated.

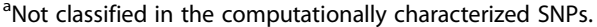

identified in rice cvs. showing high level of $W x$ transcripts (Cai et al. 1998) was highlighted at position +272 in the rice accessions Alinano $\mathrm{C}$ and Zhen Shang 47. In these two accessions, also a 1 bp deletion was present in intron 1 at position -862 and a 3 bp deletion in intron 10 at 2,241 bp from the start codon (data not shown).

\section{Generation of new molecular markers from allele mining}

With the aim of identifying more informative markers allowing a better discrimination between accessions with AAC higher than $25 \%$ from those with lower levels, one and three SNPs were selected from Antoni/Gigante Vercelli and Alinano C/Zhen Shang 47, respectively, for molecular markers development. dCAPS molecular markers were obtained from the SNPs identified at positions $-1,514$ (promoter region) (Antoni and Gigante Vercelli), $+1,801$ (exon 9), $+2,282$ (intron 10) and $+2,806$ (intron 12) (Alinano $C$ and Zhen Shang 47) and used to screen the 127 accessions (Figure 3; Table 1). Antoni and Gigante Vercelli haplotype $(\mathrm{T})$ at position $-1,514$ was identified in 17 additional accessions belonging to the group with AAC 20.65 - 24.85\%. Among them, 11 carried the C allele for the SNP in exon 6 as Antoni and Gigante Vercelli. Considering both the SNPs, four allelic patterns associated to different levels of AAC were identified (Table 5). The AG allele (the first base referred to the SNP in exon 6, while the second to position -1,514) was present in 89 accessions and associated to a mean AAC value of $18.99 \%$ (without considering the waxy variety Calmochi 101); the GC allele was found in 18 accessions with a mean AAC value of $23.50 \%$; the AT allele, carried by 6 accessions, corresponded to an AAC average of $24.24 \%$; the last allele, CT, was identified in 13 genotypes with a mean AAC of $22.75 \%$.

Considering the RM190 alleles associated to the four haplotypes identified for exon 6 and the SNP at $-1,514$, it was observed that the combination of $\mathrm{CT}_{20}$ with $\mathrm{A}$ (exon 6) and G (SNP -1,514) was always associated to an AAC higher than 24.5\% (Table 1), thus providing a previously un-indentified tool for selecting rice accessions with high AAC. Allelic variation observed for the SNPs at positions $+1,801,+2,282$ and $+2,806$ can finally provide useful diagnostic tools for selecting accessions with AAC higher than $24 \%$ when specific donors like Merlè, CNA 4081, Orione, Zhen Shang 47 and Alinano $\mathrm{C}$ are used (Table 1); for these accessions, in fact, a unique CGT allelic pattern was observed for the three SNPs. The SNP at position $-1,514$ slightly increased the AAC explained variation to $80.1 \%$ when considering the SNPs only, and to $80.3 \%$ when the RM190 was included (Table 3). However, when only the models having all the variables significant at $\mathrm{P} \leq 0.05$ were considered, the model with the highest ability to explain AAC variation (79.5\%) was the one with SNPs at intron1 and exon 6 


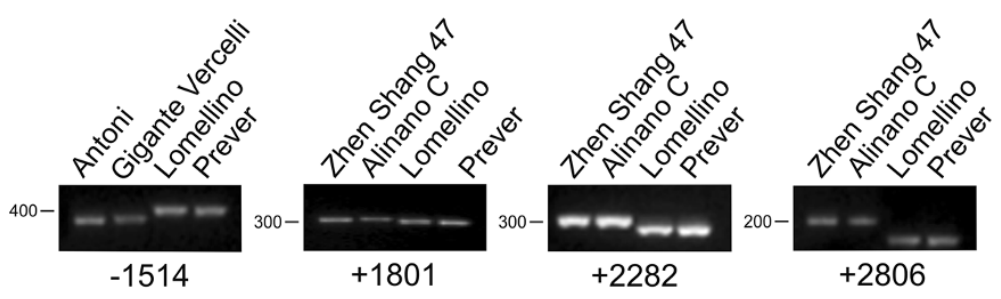

Figure 3 dCAPS molecular markers developed from SNPs found in Antoni, Gigante Vercelli, Alinano C and Zhen Shang 47 Waxy alleles. Lomellino and Prever were used as control. Primers and restriction enzymes for each molecular marker are listed in Additional file 1: Table S1.

(Table 3), a result which is fully in agreement with previous work (Caffagni et al. 2013).

\section{Relationships between grain shape parameters and Waxy haplotypes}

Correlation analyses between grain shape parameters for the 126 non-glutinous rice accessions (Table 1) are indicated in Figure $4 \mathrm{~A}, \mathrm{~B}$ and showed a close agreement with those reported by Tran et al. (2012). The most obvious correlations were between the L/W ratio and either grain length (positive) or grain width (negative). A negative correlation exists between the length and the width of the grain while regarding $\mathrm{AAC}$, grain length and the L/W ratio were positively correlated with it, whereas grain width was negatively correlated. An analysis of the histogram distribution plots of the variables indicated that the distributions of AAC and grain width clearly showed two modes (Figure 4 A).

In rice, traits related to grain size, shape and cooking properties have a large impact on market appreciation and play a pivotal role in the adoption of new varieties (Webb 1991; Juliano 2003). It is therefore interesting to note that, as shown in Table 6, we found some surprising associations between the haplotypes at the waxy locus and the shape of the rice caryopsis. In particular, the SNP at the first intron (which identifies $W x^{a}$ and $W x^{b}$ genotypes) was associated with differences in the width of the grain, the length to width ratio (L/W), and the length of the kernel. The SNP at the sixth intron showed no association with these traits by itself, but it

Table 5 Apparent Amylose Content (AAC) values observed in accessions with different allelic patterns for the newly identified SNP at position -1,514 in Antoni and Gigante Vercelli associated to the exon 6 SNP

\begin{tabular}{llll}
\hline $\begin{array}{l}\text { Allelic } \\
\text { pattern }^{\text {a }}\end{array}$ & $\begin{array}{l}\text { Number of } \\
\text { accessions }\end{array}$ & AAC range (\%) & $\begin{array}{l}\text { Mean of } \\
\text { AAC (\%) }\end{array}$ \\
\hline GA & 89 & $14.92-23.27$ & 18.99 \\
GC & 18 & $22.21-25.03$ & 23.50 \\
TA & 6 & $23.57-24.85$ & 24.24 \\
TC & 13 & $20.65-23.93$ & 22.75 \\
\hline
\end{tabular}

${ }^{a}$ The two bases indicate the SNP alleles at position $-1,514$ and in exon 6 , respectively. slightly increased the overall explained variance once the SNP at the first intron was considered. This suggests that the latter SNP was the actual responsible of the association, whereas the SNP at the intron 6 has only an ancillary effect. Even the $\mathrm{CT}_{\mathrm{n}}$ showed a significant association, but its explanatory capability of the variance of grain biometric parameters was lower than that of the SNP at the first intron, thus that, given it also has no

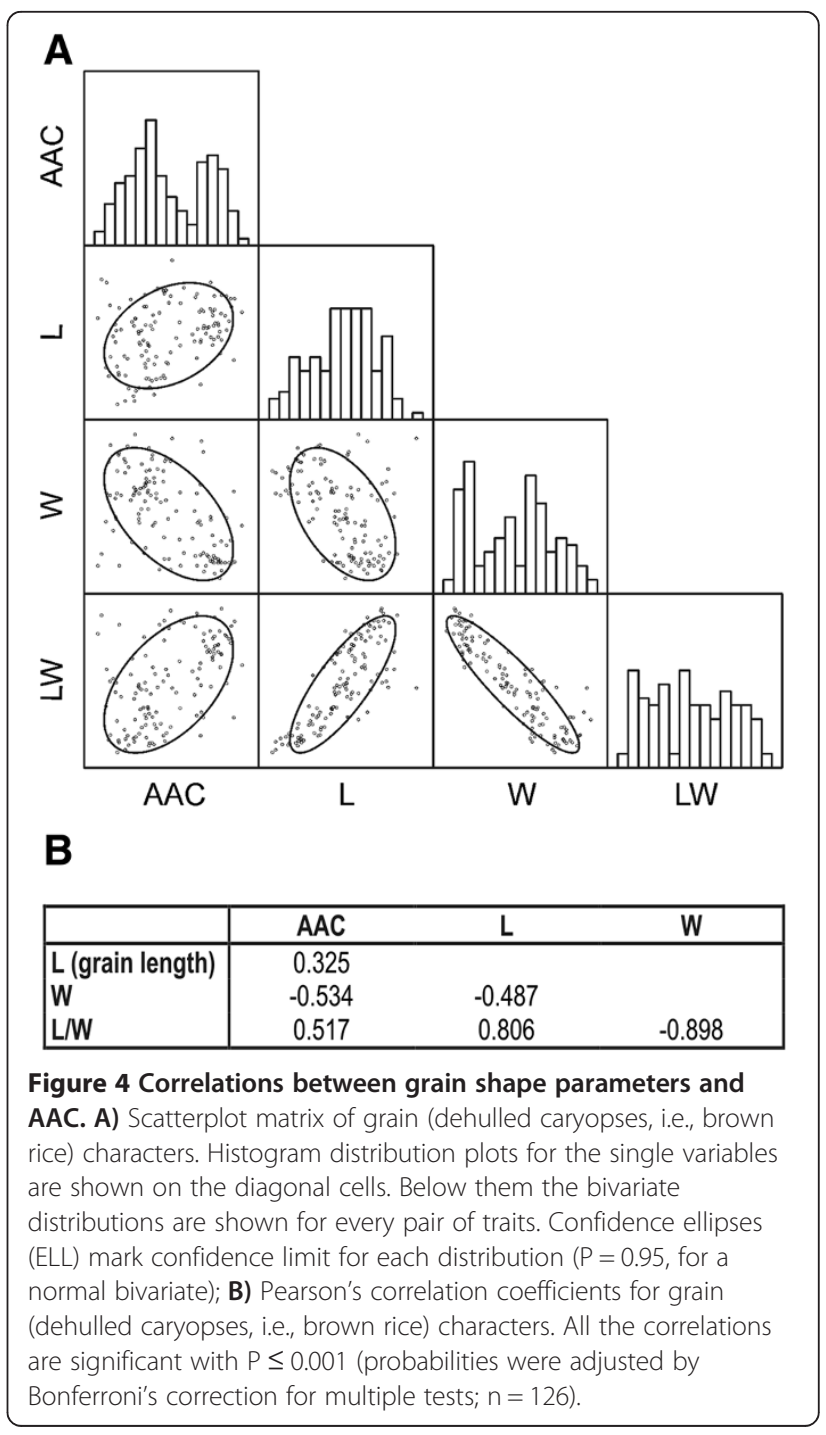


Table 6 Percentage of variation for seed biometric indexes explained by the tested GBSSI molecular markers in the germplasm collection

\begin{tabular}{|c|c|c|c|c|c|c|}
\hline \multirow[b]{2}{*}{ Molecular marker } & \multicolumn{2}{|c|}{ Decorticated grain lenght } & \multicolumn{2}{|c|}{ Decorticated grain width } & \multicolumn{2}{|c|}{ Lenght to width ratio } \\
\hline & Variation (\%) & p-value & Variation (\%) & p-value & Variation (\%) & p-value \\
\hline SNP intron1 & 30.8 & 0.000 & 49.7 & 0.000 & 47.7 & 0.000 \\
\hline $\mathrm{CTn}$ & 10.8 & 0.136 & 37.7 & 0.000 & 30.2 & 0.000 \\
\hline \multirow[t]{2}{*}{ SNP intron1/SNP exon6 } & 30.9 & 0.000 intron 1 & 52 & 0.000 intron 1 & 49.3 & 0.000 intron 1 \\
\hline & & 0.791 exon6 & & 0.051 exon6 & & 0.11 exon6 \\
\hline \multirow[t]{2}{*}{ SNP intron1/CTn } & 21.8 & $0.043 \mathrm{CTn}$ & 37.7 & $0.008 \mathrm{CTn}$ & 33.7 & $0.033 \mathrm{CTn}$ \\
\hline & & 0.000 intron 1 & & 0.798 intron 1 & & 0.015 intron 1 \\
\hline \multirow[t]{3}{*}{ SNP intron1/CTn/SNP exon6 } & 22.8 & 0.000 intron 1 & 38.3 & 0.41 intron 1 & 35.4 & 0.003 intron 1 \\
\hline & & $0.029 \mathrm{CTn}$ & & $0.020 C T n$ & & $0.029 \mathrm{CTn}$ \\
\hline & & 0.243 exon6 & & 0.295 exon6 & & 0.087 exon6 \\
\hline \multirow[t]{3}{*}{ SNP intron1/SNP exon6/SNP intron6 } & 10.2 & 0.007 intron 1 & 29.2 & 0.000 intron 1 & 26.0 & 0.000 intron 1 \\
\hline & & 0.779 exon6 & & 0.045 exon6 & & 0.102 exon6 \\
\hline & & 0.363 intron6 & & 0.055 intron6 & & 0.098 intron6 \\
\hline \multirow{4}{*}{$\begin{array}{l}\text { SNP intron1/SNP exon6/SNP } \\
\text { intron6/CTn }\end{array}$} & 22.9 & 0.019 intron 1 & 39.3 & 0.465 intron 1 & 35.6 & 0.316 intron 1 \\
\hline & & 0.244 exon6 & & 0.293 exon6 & & 0.088 exon6 \\
\hline & & 0.627 intron6 & & 0.188 intron6 & & 0.566 intron6 \\
\hline & & $0.037 \mathrm{CTn}$ & & $0.037 C T n$ & & $0.064 \mathrm{CTn}$ \\
\hline
\end{tabular}

For each marker the explained variation and the associated p-value considering each molecular marker singularly or in association with others are reported.

direct effect on the functionality of GBSSI, its association is most probably indirect and most likely ascribable to the phylogenetic association of some $\mathrm{CT}_{n}$ haplotypes with the two versions of the SNP at the first intron.

The positive correlation between AAC and L/W ratio, and the negative one with the width of the grain were further analysed (Figure 5). In fact, by plotting L/W ratio versus AAC for the overall genotype set used in this work, the positive correlation $(\mathrm{r}=0.517, \mathrm{P}<0.001)$ is immediately apparent (Figure 5A). When the genotype set is grouped according to the haplotype at the SNP at intron $1(\mathrm{G} / \mathrm{T})$, that is, the $W x^{a} / W x^{b}$ allelic version is superimposed onto the correlation plot, it becomes evident that: (a)- this SNP offers a sharp distinction between genotypes with $\mathrm{AAC} \leq 21 \%\left(W x^{b}\right)$ and $\mathrm{AAC} \geq 21 \%$ $\left(W x^{a}\right)$; (b)- in our set of genotypes, there seems to be a break for AAC around 21.5\%, which, therefore, appears to be a more precise threshold value for discriminating between $W x^{a} / W x^{b}$ allelic versions, even though a few genotypes spill over, and small differences in AAC can actually occur because of the method of assay (Fitzgerald et al. 2009); (c)- the frequencies of genotypes having an intermediate Apparent Amylose Content (haplotype $W x^{b}$ ) are skewed towards low L/W ratios, and, vice-versa, the frequencies of high amylose genotypes (haplotype $W x^{a}$ ) are skewed towards high $\mathrm{L} / \mathrm{W}$ ratios. It is indeed the presence of these skewed distributions that generates the positive correlation between $\mathrm{L} / \mathrm{W}$ ratio and $\mathrm{AAC}$, and, then, the association between $\mathrm{L} / \mathrm{W}$ ratio and the SNP at the first intron.

Furthermore, the inverse correlation between AAC and grain width is slightly stronger than that between AAC and L/W (Figure 4B; Tran et al. 2012). Correspondingly, grain width has a slightly higher explanatory ability on AAC than L/W ratio (Figure $5 \mathrm{~B}$ ). This is due to the stronger skewing of the grain width distribution within the $W x^{a}$ haplotype, which, in turn, is linked to the up-mentioned presence of two clearly distinct modes in grain width (Figure 4A): one, at $2.2 \mathrm{~mm}$, typifies $W x^{a}$ genotypes, while the other, around $3 \mathrm{~mm}$, characterizes $W x^{b}$ genotypes (Figure $5 \mathrm{~B}$ ). In the case of width, $W x^{a}$ genotypes are more sharply crowded close to their mode than what occurs for the L/W ratio, that is, their frequencies are more skewed towards thinner grains. This makes the existence of two different modes, one for each $W x$ haplotype, immediately evident even in the overall population (Figure 4A), and also augments the slope of the regression line (Figure $5 \mathrm{~B}$ ), thus that the effect of the $W x$ haplotype upon grain width appears to be greater than that on $\mathrm{L} / \mathrm{W}$ ratio.

Interestingly, $\mathrm{AAC}$ also positively correlates with cycle length $(r=0.378, P<0.001$; data for 124 non-glutinous rice accessions for which maturity data were available; Table 1), whereas no significant correlation was observed between cycle length (days to maturity) and grain shape 

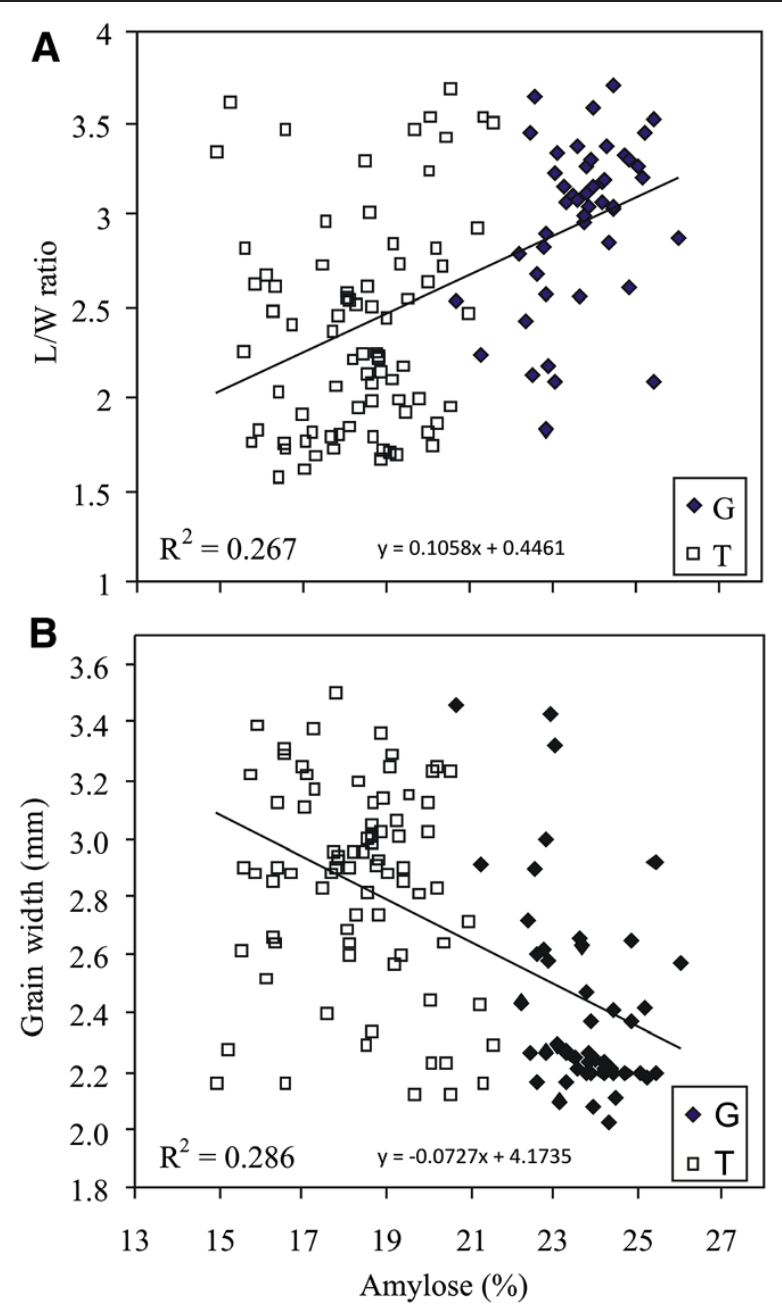

Figure 5 Relationships between $A A C$ and $L / W$ ratio correlations. Scatterplots of: L/W ratio versus AAC (A), and grain width versus AAC (B). Datapoints for dehulled caryopses of 126 non glutinous cultivars, grouped according to the haplotype at the SNP of intron 1 (47 accessions with $\mathrm{G}$ allele; 79 accessions with $\mathrm{T}$ allele). The regression line for the whole dataset and its determination coefficient are shown in each plot.

parameters (data not shown). The relationship would appear to be owed to a preponderance of long-cycle accessions in the high-amylose $W x^{a}$ group (data not shown). It is however not a necessary correlation, since some genotypes with high AAC and short cycle were present as well (Gladio and Sandora; Table 1). For the same reason, this should not be an environmentally-caused relationship, although the amylose content can be affected by the temperature during grain ripening (Gomez 1979).

\section{Discussion}

\section{Allelic analyses of the Waxy gene}

Different alleles of the Waxy gene in rice are defined by a relatively small number of molecular markers. The RM190 $\mathrm{CT}_{\mathrm{n}}$ itself could explain more than $80 \%$ of
Apparent Amylose Content variation in several different rice collections (Shu et al. 1999; Bergman et al. 2001; Tan and Zhang 2001; Dobo et al. 2010). According to other works, for our germplasm collection RM190 could explain a high percentage of variation in AAC (74.5\%), considering non-glutinous accessions. Dobo and Coworkers (2010) found that the $\mathrm{CT}_{20}$ allele was associated to high or intermediate AAC for European or US cvs., respectively; similarly, in our analyses, this allele was always associated to AAC higher than $22 \%$ but regardless to the origin of the genotype. As previously observed (Bergman et al. 2001; Tan and Zhang 2001; Dobo et al. 2010), accessions with a number of CT repeats ranging from 17 to 19 showed an AAC variable from 15 to $23 \%$. This behaviour is in agreement to the fact that the RM190 microsatellite is only a molecular marker associated to the Waxy gene without a functional role in determining the level of amylose in rice seeds (Chen et al. 2008; Dobo et al. 2010).

A functional role was instead characterized for the G/T SNP in the donor splicing site of the leader intron. In literature the $\mathrm{G}$ haplotype, which characterises the $W x^{a}$ allele, is associated to high AAC, while the T allele $\left(W x^{b}\right)$ to low and intermediate AAC (Shu et al. 1999; Bergman et al. 2001; Tan and Zhang 2001; Dobo et al. 2010). For our non-glutinous germplasm, the $\mathrm{G}$ haplotype was always associated to AAC higher than $22 \%$. Considering the high level of AAC variation explained (77.5\%) and the observation that only a very small increase in explained AAC variation resulted from adding the RM190 data, we concluded that our results are in accordance with the SNP in intron 1 as being the main determinant affecting amylose amount in endosperm.

Several researches have been focused in identifying different $W x$ alleles associated to the wide variability in AAC. Larkin and Park (2003) found two SNPs, an A/C in exon 6 and a $\mathrm{C} / \mathrm{T}$ in exon 10, causing non-conservative amino acid substitutions at the protein level which could change the specific activity of the GBSSI protein. Together with the SNP in intron 1, these polymorphisms explained 93.8\% of ACC variation in European and US germplasm collections (Dobo et al. 2010). Chen et al. (2008) screened 171 rice accessions originating from 43 countries for the above mentioned GBSSI SNPs and found that the majority of cvs. with an AAC ranging from 21 to $22 \%$ carried the haplotype $\mathrm{C}$ at exon 6 . Results with our collection highlighted no variations for exon 10 and an association of the SNP in exon 6 to AAC values from 21 to $24 \%$ for the $\mathrm{C}$ allele, while the A allele was present in accessions with AAC ranging from waxy to $21 \%$ and from 24 to $26 \%$. The three haplotypes defined by the combination of the two SNPs in intron 1 and exon 6 were able to explain the $79.5 \%$ of AAC variation in non-glutinous accessions. Even in this case, when the 
RM190 was added to statistical analyses it did not show a significant effect on the percentage of explained AAC variation, confirming that this molecular marker does not have a direct effect on the functionality of the enzyme (Dobo et al., 2010). These results underline that the two SNPs (intron 1 and exon 6) represent the more suitable tools to be used in breeding programs for our germplasm collection and that $a$ priori generalization of the efficiency for molecular markers in predicting AAC values could represent a risky procedure.

A higher level of AAC variation was found to be explained in previous work with respect to the ones observed here, even when comparisons were based on the same molecular markers. Considering that our collection is represented by genotypes originated from a wide range of countries, it is possible that the genetic diversity to be explained was greater than in other studies where the germplasm originated from more restricted areas: US (Ayres et al. 1997; Bergman et al. 2001), Europe and US (Dobo et al. 2010), China (Tan and Zhang 2001) and Korea (Shu et al. 1999).

\section{Identification of new molecular markers associated to apparent Amylose content}

To date, most of the studies on the topic have been based on the characterization of molecular markers associated to waxy and low AAC (Isshiki et al. 1998; Larkin and Park 2003; Wanchana et al. 2003; Mikami et al. 2008; Liu et al. 2009; Tran et al. 2012). However the preference for rice with high $\mathrm{AAC}$ in occidental countries underlines the need of developing new markers facilitating the identification of genotypes with high AAC. A sub-sample of 21 genotypes belonging to our collection and representing four AAC classes was selected for the GBSSI gene re-sequencing. A very high level of sequence identity was observed and most of the identified polymorphisms were detected in non-coding regions. Thus, the GBSSI gene has been likely conserved during evolution as its key role in controlling the accumulation of amylose in caryopsis.

As previously reported, the amount of amylose in endosperm is principally related to the post-transcriptional regulation of the Waxy gene which is influenced by the intron 1 SNP (Wang et al. 1995; Bligh et al. 1998; Cai et al. 1998). However, our sequencing comparisons revealed that genotypes belonging to different AAC classes showed the same GBSSI haplotypes and alleles suggesting that other genes involved in starch synthesis can affect the amylose content in endosperm. These additional genes could be represented by the $D u$ gene, encoding for a Starch Synthase enzyme (Zeng et al. 2007), the Shr gene encoding for a Sucrose Synthase (Kawagoe et al. 2005), or other genes encoding for enzymes involved in starch metabolism such as ADP glucose pyrophosphorylase (Venu et al. 2011) and $\alpha$-glucan phosphorylase (Satoh et al. 2008).

Antoni and Gigante Vercelli accessions carried the SNP in exon 6 typical of the $W x^{i n}$ allele, the SNP in intron 1 (position -399) and one in the promoter region (position -1,514). Moreover, two accessions with high AAC, Zhen Shang 47 and Alinano C, showed the highest sequence variation with respect to Nipponbare that included a non-conservative SNP in the coding region of exon 9, causing a Pro to Ser substitution in the GBSSI protein. As this mutation implicates the substitution of an apolar amino acid to a polar one it should be possible that it increases the activity of the GBSSI enzyme, even if its effect should be small as the SNP does not affect the AAC variation significantly (Tab. 3 ).

New dCAPS markers were developed exploiting the SNPs in intron 1 at position -1,514 (identified in Antoni and Gigante Vercelli), exon 9, intron 10 and intron 12 (identified in Zhen Shang 47 and Alinano C). After screening of the 127 accessions with these molecular markers it was observed that the combination of the $\mathrm{CT}_{20} \mathrm{RM} 190$ alleles in combination with the A haplotype for exon 6 and the $\mathrm{G}$ haplotype for SNP -1,514 was always associated to an AAC higher than $24.5 \%$ thus providing an efficient tool for selecting high AAC rice accessions.

\section{Associations between molecular markers and grain shape parameters}

The results presented in this work confirm general observations (Webb 1991; Juliano 2003) that high AAC is associated with a slender grain, whereas rices having an intermediate amylose content most often have bold grains. We clearly highlighted that the frequencies of genotypes having a low AAC $(<21.5 \%)$ are skewed towards low L/W ratios while the frequencies of intermediatehigh amylose genotypes ( $>21.5 \%$ AAC) are skewed towards high $\mathrm{L} / \mathrm{W}$ ratios. These skewings can be imputed to selection for consumer preferences: for cooking and processing, rice are conventionally classified as short-, medium- and long-grain types, and an $\mathrm{AAC} \geq 20 \%$ is preferred for long-grain rice, whereas an AAC $<20 \%$ is commonly favoured for short- and medium-grain types (Webb 1991). The width of the grain is as much, or even more, linked to the cooking application of rice than the $\mathrm{L} / \mathrm{W}$ ratio: long, thin-grain rice is typically used in applications requiring distinct shape and texture, and high amylose ensures that cooked grains are firm and remain separate; on the other hand, medium- and short-grain rice are ideal for puddings, desserts, and similar applications, and low amylose content allows to obtain cooked grains that are soft, moist and sticky. Grain width and $\mathrm{L} / \mathrm{W}$ ratio are more distinctive of the cooking application than grain length because long-grain rice with bold kernel are typically used to prepare 'risotto', and 
many of them are traditional varieties with intermediate AAC. In fact, the EU classification (see Ferrero and Nguyen, 2004 for a synoptic table of USA and EU grades) further distinguishes long-grain rice (length > $6.0 \mathrm{~mm})$ into long $\mathrm{A}(2<\mathrm{L} / \mathrm{W}<3)$ and long $\mathrm{B}$ types $(\mathrm{L} / \mathrm{W} \geq 3)$. The former wide-grain type is used for 'risotto' and often has intermediate $\mathrm{AAC}$, while the latter thin-grain type includes high amylose rice for oriental and side dishes, prepared entrees, rice salad and garnitures. Thus, grain width is more related to cooking applications than grain length, and then it has a better association with AAC. Further work is crucial to characterize the genetic basis of the two different modes observed for grain width in $W x^{a}$ and $W x^{b}$ haplotypes, and many candidate genes are available (Huang et al. 2013).

It can be worthy to note that the positive correlation between AAC and cycle length may occur since the high-amylose $W x^{a}$ haplotype, primarily found in the indica subspecies (Hirano and Sano 1991; Dobo et al. 2010), was most probably introduced into the japonica subspecies, which till to some decades ago lacked cultivars with high amylose (Juliano 1979), from indica genotypes, which frequently have longer cycles. It can be supposed that breeding for high AAC japonica cultivars has then been commonly done by crossing within a restrict group of late-maturing materials of indica derivation, thus that the long cycle would be an instance of genetic drift (or draft). On the other hand, the fact that no significant correlation was observed between cycle length and grain shape parameters suggests that breeding for both early and late cultivars within each of the grain-type varietal groups has broken up any hitchhiking effect between these traits. The strong association between the SNP at the first intron (which identifies $W x^{a}$ and $W x^{b}$ genotypes) and the width of the grain, the length to width ratio $(\mathrm{L} / \mathrm{W})$, and the length of the kernel is owed to the skewing of genotype frequencies towards a bold grain in $W x^{a}$ rice and towards a slender grain in $W x^{b}$ ones (Figure 5), and hence it is most likely ascribable to human selection. Simultaneous selection for two traits can increase the frequency of alleles that affect both traits favourably and leave the frequency of alleles that affect one trait favourably and one trait unfavourably at lower levels (Bennett and Swiger 1980), which is precisely what is observed in Figure 5. Selection for optimal character combinations, generating genetic correlation between suites of linked traits, is also known as correlational selection (Sinervo and Svensson 2002). Together with selection for non-shattering grains, reduced seed dormancy, whitish kernels and aroma (McCouch et al. 2012; Sang and Ge 2013), correlational selection for grain shape and AAC has marked the evolution of the rice crop according to human preferences for grain characteristics.

\section{Conclusions}

The available molecular markers utilized for evaluation of AAC classes did not provide tools for predicting accessions with AAC higher than 24.5\%. New SNPs were identified through re-sequencing of the Waxy gene and $1 \mathrm{kbp}$ of the upstream region. The related dCAPS markers increased the AAC explained variation and allowed the identification of a haplotype almost unequivocally associated to AAC higher than 24.5\%, which represent the AAC class preferred in the occidental countries markets. The SNP at the first intron, which identifies the $W x^{a}$ and $W x^{b}$ alleles, was associated with differences in the width of the grain, the L/W ratio and the length of the kernel, most likely as a result of human selection.

\section{Methods}

\section{Plant material and phenotyping for AAC and biometric} parameters

An Italian Oryza sativa L. collection of 127 accessions was used in the present study and included 40 national rice varieties belonging to the japonica ssp. and 87 foreign accessions (6 from Spain, 6 from France, 29 from the US, 10 from Portugal, 7 from Argentina and 29 from other countries worldwide), of which 125 were japonica and 2 indica. The Italian rice varieties were selected because of their past and present relevance for breeding programs, and their contribution to rice production in the last decades. The 87 foreign accessions were selected as being relevant to Italian rice breeding, or considered reference varieties at the international level.

Each rice accession was grown in triplicate field trials for two years. Maturity time of each field trials was recorded when at least $50 \%$ of the plants was ready for harvesting. Data was represented by the mean value of three replicates and indicates the days from sowing to panicles maturation. Harvested rice seeds were used for amylose quantification and biometric parameters evaluations. According to the protocol of Williams et al. (1958), with modifications by Inatsu (1988), the AAC of milled grain was measured with a FOSS FIAstar 5000 auto-analyzer which is based on a flow injection of a solution of $\mathrm{NaOH} 0.09 \%$ to the sample, the addition of an iodine solution and the spectrometric determination of the absorbance of the formed color at $720 \mathrm{~nm}$. The calibration was performed measuring the absorbance of standard rice samples carrying $15.40 \%, 23.10 \%$ and $27.7 \%$ of AAC, respectively, using a white reference. These reference samples were supplied and certified by FOSS as having their amylose content determined against amylose/ amylopectin standards. The SoFIA software (FOSS) was used to build up the calibration curve and to obtain the percentage of amylose in our samples. Each reference analysis was repeated twice for each sample. Biometric parameters of rice seeds (decorticated grain length and 
decorticated grain width) were evaluated trough optical scanner-produced high resolution images analyzed with the WinSEEDLE 2011a software (Regent Instruments Inc.).

\section{Molecular markers analyses}

To obtain genomic DNA from the rice accessions, seeds were germinated in petri dishes at $30^{\circ} \mathrm{C}$ and one-week old seedlings were transplanted and grown in a greenhouse until three leaf stages; leaves were therefore collected, frozen in liquid nitrogen and store at $-80^{\circ} \mathrm{C}$. Genomic DNA was extracted on plates using the Wizard ${ }^{\circledR}$ Magnetic 96 DNA Plant System (Promega) according to manufacturer's instructions. The CTAB DNA extraction method (Doyle and Doyle 1987) was instead applied for the 21 genotypes selected for GBSSI gene re-sequencing.

The RM190 CT repeat was assayed using the M13tailed forward primer RM-190 F (CACGACGTTGTAA AACGACCTTTGTCTATCTCAAGACAC) and the reverse primer RM-190R (TTGCAGATGTTCTTCCTGATG) (Ayres et al. 1997; Chen et al. 2008). PCR reactions were performed in $10 \mu \mathrm{l}$ containing $15 \mathrm{ng}$ of genomic DNA, $0.1 \mu \mathrm{M}$ of RM-190 F, $1 \mu \mathrm{M}$ of RM-190R and FAM-labelled M13 (CACGACGTTGTAAAACGAC), $0.2 \mathrm{mM}$ dNTPs and $1 \mathrm{U}$ GoTaq DNA Polymerase (Promega). DNA was amplified using a touchdown program as follows: denaturation at $94^{\circ} \mathrm{C}$ per $3 \mathrm{~min}, 20$ cycles at $94^{\circ} \mathrm{C}$ per $45 \mathrm{sec}$, from $61^{\circ} \mathrm{C}$ to $51.5^{\circ} \mathrm{C}$ per $45 \mathrm{sec}$, reducing the annealing temperature of $0.5^{\circ} \mathrm{C}$ for each cycle, and $72^{\circ} \mathrm{C}$ per $45 \mathrm{sec}, 24$ cycles at $94^{\circ} \mathrm{C}$ per $45 \mathrm{sec}, 51^{\circ} \mathrm{C}$ per $45 \mathrm{sec}$ and $72^{\circ} \mathrm{C}$ per $45 \mathrm{sec}$ and a final extension of $72^{\circ} \mathrm{C}$ per $10 \mathrm{~min}$. Labelled PCR products were run in a 3130 Genetic Analyzer (Applied Biosystems). ROX size standard (Applied Biosystems) was used.

PCRs for dCAPS analyses of the G/T polymorphism in intron 1 were carried out in $20 \mu \mathrm{l}$ using GoTaq DNA Polymerase (Promega) supplemented with 5\% DMSO and with $20 \mathrm{ng}$ of genomic DNA, $1 \mu \mathrm{M}$ of GBSS-W2F, $1 \mu \mathrm{M}$ of GBSS-W2R (Ayres et al. 1997; Chen et al. 2008). Amplification conditions were: $94^{\circ} \mathrm{C}$ per $4 \mathrm{~min}$ followed by 40 cycles of $94^{\circ} \mathrm{C}$ per $40 \mathrm{sec}, 60^{\circ} \mathrm{C}$ per $50 \mathrm{sec}$ and $72^{\circ} \mathrm{C}$ per 1 min per kb and a final extension of $72^{\circ} \mathrm{C}$ per $10 \mathrm{~min}$. After amplification $5 \mu \mathrm{l}$ of PCR products were digested with $1 \mathrm{U}$ of $A c c I$ restriction enzyme (New England BioLabs) in a total volume of $10 \mu \mathrm{l}$ at $37^{\circ} \mathrm{C}$ over night. The samples were run on $2 \%$ agarose gel. Each digested sample was compared alongside with its notdigested cognate control.

The presence of $W x^{o p}, W x^{i n}, W x^{m q}, W x^{h p}$ and $w x$ alleles and the variability for the SNP in exon 10 identified by Larking and Park (2003) were assessed in our collection through the sequencing of exons 4, 6, 5, 2 and 10 using the same procedure for the allele mining experiment described below.
To detect the SNPs at position $-1,514,+1,801,+2,282$ and $+2,806$ by dCAPS analysis, mismatched forward primers were designed by dCAPS Finder 2.0 software (http://helix.wustl.edu/dcaps/dcaps.html). Primers and restriction enzymes for dCAPS assay are listed in Additional file 1: Table S1. For PCR amplification, the same protocol used for detecting the G/T polymorphism in the leader intron was used.

\section{Allele mining}

For the allele mining of the GBSSI alleles in the 21 selected genotypes, six overlapping regions, ranging from $800 \mathrm{bp}$ to 2,500 bp, were PCR amplified from genomic DNA with the same protocol described above for the G/T polymorphism. Primers designed on the Nipponbare genomic sequence (Genebank AC NC_008399; Additional file 2: Table S2) were utilized for genomic DNA amplifications using the combinations indicated in Additional file 2: Table S2. The amplified regions covered the entire gene plus $1 \mathrm{kbp}$ of the upstream putative regulatory region. After gel purification by the Wizard ${ }^{\circledR} \mathrm{SV}$ Gel and PCR Clean-Up System (Promega), PCR products were directly sequenced. Sequencing reactions were accomplished by the use of ABI BigDye Terminator version 3.1 (Applied Biosystem) in forward and reverse directions with $5 \mu \mathrm{l}$ of each PCR amplification product and the same primers used for PCR amplifications or internal primers. All primers were designed using the Primer3 0.4.0 software (http://frodo.wi.mit.edu/) and blasted against the rice genomic sequence on the Gramene website (http:// www.gramene.org) to ensure the specificity for the GBSSI gene.

\section{Computational analyses}

The RM190 microsatellite was analyzed using the GeneMapper software (Applied Biosystem). Sequence assembly was assessed with the ContigExpress tool of Vector NTI Software (Invitrogen) using the Nipponbare genomic sequence as reference. Sequence comparison was carried out by MultAlin software (http://multalin.toulouse.inra.fr/multalin/). All data were analysed with the Systat 12 software (SPSS Inc., Chicago, IL, USA). The relationships between numerical variables (AAC and grain biometrical characters) were evaluated by Pearson correlation coefficients and regression analysis. The associations between numerical variables (AAC) and categorical variables (marker haplotypes) were analysed according to the General Linear Model (GLM) procedure.

\section{Additional files}

Additional file 1: Table S1. Markers obtained from the newly discovered SNPS. For each marker, primers, SNP position, restriction 
enzyme and origin of the restricted amplicon are indicated. The mutated base is in bold and underlined.

Additional file 2: Table S2. Primer combinations used to amplify the Waxy alleles and internal primers used for amplicons sequencing.

\section{Competing interests}

The authors declare that they have no competing interests.

\section{Authors' contributions}

$\mathrm{CB}$ carried out the molecular genetic studies, the sequence alignment and drafted the manuscript; DC, RP, PB, SU, GO, FD participated to molecular genetic studies, the sequence alignment and determination of Apparent Amylose Content; AG participated in data analysis and helped to draft the manuscript; EL, LC participated in the design of the study and helped to draft the manuscript; GV designed the study and helped to draft the manuscript. All authors read and approved the final manuscript.

\section{Acknowledgements}

This study was funded by grants from AGER Foundation, (RISINNOVA project grant n. 2010-2369) and MiPAAF (POLORISO project).

\section{Author details}

${ }^{1}$ Rice Research Unit, CRA-Consiglio per la Ricerca e la Sperimentazione in Agricoltura, S.S. 11 to Torino, Km 2,5, 13100 Vercelli, Italy. ${ }^{2}$ Genomics Research Centre, CRA-Consiglio per la Ricerca e la Sperimentazione in Agricoltura, Via S. Protaso 302, 29017 Fiorenzuola d'Arda, Piacenza, Italy. ${ }^{3}$ Department of Plant Biology and Crop Production, CRA-Consiglio per la Ricerca e la Sperimentazione in Agricoltura, Roma, Italy.

\section{Received: 31 October 2013 Accepted: 26 December 2013}

\section{Published: 2 January 2014}

\section{References}

Asaoka M, Okuno K, Fuwa H (1985) Effect of environmental-temperature at the milky stage and amylose content and fine-structure of amylopectin of waxy and non-waxy endosperm starches of rice (Oryza sativa L.). Agric Biol Chem 49:373-379

Ayres NM, McClung AM, Larkin PD, Bligh HFJ, Jones CA, Park WD (1997) Microsatellites and a single-nucleotide polymorphism differentiate apparent amylase classes in an extended pedigree of US rice germplasm. Theor Appl Genet 94:773-781

Bao JS, Corke H, Sun M (2006) Nucleotide diversity in starch synthase lla and validation of single nucleotide polymorphisms in relation to starch gelatinization temperature and other physicochemical properties in rice (Oryza sativa L.). Theor Appl Genet 113:1171-1183

Bennett GL, Swiger LA (1980) Genetic variance and correlation after selection for two traits by index, independent culling levels and extreme selection. Genetics 94:763-775

Bergman CJ, Delgado JT, McClung AM, Fjellstrom RG (2001) An improved method for using a microsatellite in the rice waxy gene to determine amylose class. Cereal Chem 78:257-260

Bligh HFG, Till RJ, Jones CA (1995) A microsatellite sequence closely linked to the Waxy gene of Oryza sativa. Euphytica 86:83-85

Bligh HFJ, Larkin PD, Roach PS, Jonea PA, Fu H, Park WD (1998) Use of alternate splicing sites in granule-bound starch synthase mRNA from low-amylose rice varieties. Plant Mol Biol 38:407-415

Caffagni A, Albertazzi G, Gavina G, Ravaglia S, Gianinetti A, Pecchioni N, Milc J (2013) Characterization of an Italian rice germplasm collection with genetic markers useful for breeding to improve eating and cooking quality. Euphytica 194(3):383-399

Cai XL, Wang ZY, Xing YY, Zhang JL, Hong MM (1998) Aberrant splicing of intron 1 leads to the heterogeneous $5^{\prime}$ UTR and decreased expression of waxy gene in rice cultivars of intermediate amylose content. Plant J 14:459-465

Chen MJ, Bergman C, Pinson S, Fjellstrom R (2008) Waxy gene haplotypes: Associations with apparent amylase content and the effect by the environment in an international rice germplasm collection. J Cereal Sci 47:536-545

Chiaramonte E, Rhazi L, Aussenac T, White RD (2012) Amylose and amylopectin in starch by asymmetric flow field-flow fractionation with multi-angle light scattering and refractive index detection (AF4-MALS-RII). J Cereal Sci 56:457-463
Dobo M, Ayres N, Walker G, Park WD (2010) Polymorphism in the GBSS gene affects amylose content in US and European rice germplasm. J Cereal Sci 52:450-456

Doyle J, Doyle JL (1987) Genomic plant DNA preparation from fresh tissue - the CTAB method. Phytochem Bull 19:11

Ferrero A, Nguyen NV (2004) The sustainable development of rice-based production systems in Europe. Int Rice Comm Newsl 53:115-124

Fitzgerald MA, Bergman CJ, Resurreccion AP, Möller J, Jimenez R, Reinke RF, Martin M, Blanco P, Molina F, Chen MH, Kuri V, Romero MV, Habibi F, Umemoto T, Jongdee S, Graterol E, Reddy KR, Zaczuk Bassinello P, Sivakami R, Rani NS, Das S, Wang YJ, Indrasari SD, Ramli A, Ahmad R, Dipti SS, Xie L, Lang NT, Singh P, Toro DC, Tavasoli F, Mestres C (2009) Addressing the dilemmas of measuring amylose in rice. Cereal Chem 86(5):492-498

Gomez KA (1979) Effect of environment on protein and amylose content of rice. In: Chemical aspects of rice grain quality. International Rice Research Institute, IRRI, Los Baños, Laguna, Philippines, pp 59-68

Hirano HY, Sano Y (1991) Molecular characterization of the waxy locus of rice (Oryza sativa L.). Plant Cell Physiol 32:989-997

Huang R, Jiang L, Zheng J, Wang T, Wang H, Huang Y, Hong Z (2013) Genetic bases of rice grain shape: so many genes, so little known. Trends Plant Sc 18:218-226

Inatsu O (1988) Studies on improving the eating quality of Hokkaido Rice. Report of Hokkaido Prefectural Agric. Exp Stn 66:3-7

Isshiki M, Morino K, Nakajima M, Okagaki RJ, Wessler SR, Izawa T, Shimamoto K (1998) A naturally occurring functional allele of the rice waxy locus has GT to $\Pi \pi$ mutation at the $5^{\prime}$ splice site of the first intron. Plant J 15(1):133-138

Juliano BO (1971) A simplified assay for milled-rice amylose. J Cereal Sci 16:334-340

Juliano BO (1979) The chemical basis of rice grain quality. In: Chemical Aspects of Rice Grain Quality. International Rice Research Institute, IRRI, Los Baños, Laguna, Philippines, pp 69-90

Juliano BO (1992) Structure and function of the rice grain and its fractions. Cereal Foods World 37:772-774

Juliano BO (2003) Rice chemistry and quality. Philippine Rice Research Institute, Manila, Philippines

Kawagoe Y, Kubo A, Satoh H, Takaiwa F, Nakamura Y (2005) Roles of isoamylase and ADP-glucose pyrophosphorylase in starch granule synthesis in rice endosperm. Plant J 42:164-174

Kharabian A (2010) An efficient computational method for screening functional SNPs in plants. J Theor Biol 265:55-62

Kumar I, Khush GS (1987) Genetic-analysis of different amylose levels in rice. Crop Sci 27:1167-1172

Larkin PD, Park WD (1999) Transcript accumulation and utilization of alternate and non consensus splice sites in rice granule bound starch synthase is temperature-sensitive and controlled by a single nucleotide polymorphism. Plant Mol Biol 40:719-727

Larkin PD, Park WD (2003) Association of waxy gene single nucleotide polymorphisms with starch characteristics in rice (Oryza sativa L.). Mol Breed 12:335-339

Li Y, Shoemaker CF, Ma J, Moon KJ, Zhong F (2008) Structure-viscosity relationships for starches from different rice varieties during heating. Food Chem 106:1105-1112

Liu L, Ma X, Liu S, Zhu C, Jiang L, Wang Y, Shen Y, Ren Y, Dong H, Chen L, Liu X, Zhao Z, Zhai H, Wan J (2009) Identification and characterization of a novel Waxy allele from Yunnan rice landrace. Plant Mol Biol 71:609-626

McCouch SR, Kovach MJ, Sweeney M, Jiang H, Semon M (2012) The dynamics of rice domestication: A balance between gene flow and genetic isolation. In: Paul G, Famula TR, Bettinger RL, Brush SB, Damania AB (eds) Biodiversity in agriculture: domestication, evolution, and sustainability. Cambridge University Press, New York, USA, pp 311-329

McKenzie KS, Rutger JN (1983) Genetic analysis of amylose content, alkali spreading score and grain dimension in rice. Crop Sci 23:306-313

Mikami I, Uwatoko N, Ikeda Y, Yamaguchi J, Hirano HY, Suzuki Y, Sano Y (2008) Allelic diversification at the $w x$ locus in landraces of Asian rice. Theor App Genet 116:979-989

Park IM, Ibáñez AM, Zhong F, Shoemaker CF (2007) Gelatinization and pasting properties of waxy and non-waxy rice starches. Starch-Starke 59:388-396

Sang T, Ge S (2013) Understanding rice domestication and implications for cultivar improvement. Curr Opin Plant Biol 16, in press http://www. sciencedirect.com/science/article/pii/S1369526613000381

Sato H, Suzuki Y, Sakai M, Imbe T (2002) Molecular characterization of Wx-mq, anovel mutant gene for low-amylose content in endosperm of rice (Oryza sativa L.). Breed Sci 52:131-135 
Satoh H, Shibahara K, Tokunaga T, Nishi A, Tasaki M, Hwang SK, Okita TW, Kaneko N, Fujita N, Yoshida M, Hosaka Y, Sato A, Utsumi Y, Ohdan T, Nakamura Y (2008) Mutation of the plastidial alpha-glucan phosphorylase gene in rice affects the synthesis and structure of starch in the endosperm. Plant Cell 20:1833-1849

Shi W, Yang Y, Chen S, Xu M (2008) Discovery of a new fragrance allele ant the development of functional markers for the breeding of fragrant rice varieties. Mol Breed 22:185-192

Shu QY, Wu DX, Xia YW, Gao MW, Ayres NM, Larkin PD, Park WD (1999) Microsatellite polymorphisms on the waxy gene locus and their relationship to amylose content in indica and japonica rice, Oryza sativa L. Acta Genet Sin 26:350-358

Sinervo B, Svensson E (2002) Correlational selection and the evolution of genomic architecture. Heredity 89:329-338

Suwannaporn P, Pitiphunpong S, Champangern S (2007) Classification of rice amylose content by discriminant analysis of physicochemical properties. Starch 59:171-177

Takanokai N, Jiang H, Kubo T, Sweeney M, Matsumoto T, Kanamori H, Padhukasahasram B, Bustamante C, Yoshimura A, Doi K, McCouch S (2009) Evolutionary history of GS3, a gene conferring grain length in rice. Genetics 182:1323-1334

Tan YF, Zhang QF (2001) Correlation of simple sequence repeat (SSR) variants in the leader sequence of the waxy gene with amylose of the grain in rice. Acta Bot Sin 43:146-150

Tran DS, Tran TTH, Nguyen TLH, Ha ML, Dinh BY, Kumamaru T, Satoh H (2012) Variation on grain quality in Vietnamese rice cultivars collected from Central Vietnam. J Fac Agr Kyushu Univ 57:365-371

Venu RC, Sreerekha MV, Nobuta K, Belò A, Ning Y, An G, Meyers BC, Wang GL (2011) Deep sequencing reveals the complex and coordinated transcriptional regulation of genes related to grain quality in rice cultivars. BMC Genomics 12:190

Wanchana S, Toojinda T, Tragoonrung S, Vanavichit A (2003) Duplicated coding sequence in the waxy allele of tropical glutinous rice (Oryza sativa L.). Plant Sci 165:1193-1199

Wang ZY, Zheng FQ, Shen GZ, Gao JP, Snustad DP, Li MG, Zhang JL, Hong MM (1995) The amylose content in rice endosperm is related to the post-transcriptional regulation of the waxy gene. Plant J 7(4):613-622

Ward RM, Gao Q, de Bruyn H, Gilbert RG, Fitzgerald MA (2006) Improved methods for the structural analysis of the amylose-rich fraction from rice flour. Biomacromolecules 7:866-876

Webb BD (1991) Rice quality and grades. In: Luh BS (ed) Rice Utilization Vol. II. AVI Book Published by Van Nostrand Reinhold, New York, NY, pp 89-119

Wesley IJ, Osborne BG, Anderssen RS, Delwiche SR, Graybosch RA (2003) Chemometric localization approach to NIR measurement of apparent amylose content of ground wheat. Cereal Chem 80:462-467

Williams VR, Wu WT, Tsai HY, Bates HG (1958) Varietal differences in amylose content of rice starch. J Agric Food Chem 6(1):47-48

Zeng D, Yan W, Liu X, Qian Q, Li J (2007) Du, encoding a novel Prp1 protein regulates starch biosynthesis through affecting the splicing of $W \times b$ pre-mRNA in rice (Oryza sativa L.). Plant Mol Biol 65:501-509

doi:10.1186/1939-8433-7-1

Cite this article as: Biselli et al.: Improvement of marker-based predictability of Apparent Amylose Content in japonica rice through GBSSI allele mining. Rice 2014 7:1.

\section{Submit your manuscript to a SpringerOpen ${ }^{\circ}$ journal and benefit from:}

- Convenient online submission

- Rigorous peer review

- Immediate publication on acceptance

- Open access: articles freely available online

- High visibility within the field

- Retaining the copyright to your article

Submit your next manuscript at $\gg$ springeropen.com 